\title{
Comparative study on the antituberculous effect and mechanism of the traditional Chinese medicines NiuBeiXiaoHe extract and JieHeWan
}

Li-Yao Duan $^{1,2+}$, Yan Liang ${ }^{1 \dagger}$, Wen-Ping Gong ${ }^{1}$, Yong Xue ${ }^{1}$, Jie Mi ${ }^{1}$, Jie Wang ${ }^{1}$, Lan Wang ${ }^{1}$, Zai-Xing Jia ${ }^{1,2}$, Hong Lei ${ }^{3}$, Yu-Mei Liang ${ }^{4}$, Jun Liu' ${ }^{5}$ Yue Zheng ${ }^{1,2^{*}}$ and Xue-Qiong Wu ${ }^{1 *}$

\begin{abstract}
Background: The traditional Chinese medicine NiuBeiXiaoHe (NBXH) extract and Chinese medicine preparation JieHeWan (JHW) exhibit anti-tuberculosis effects. The anti- tuberculosis effect of NBXH was compared with that of JHW to elucidate the mechanism of action of $\mathrm{NBXH}$.

Methods: BALB/C mice aged 6-8 weeks were randomly divided into a normal control group, Tuberculosis (TB) model group, JHW treatment group, and NBXH treatment group. After 3 and 13 weeks of treatment, the therapeutic effect in each group was evaluated by comparing lung histopathology, lung and liver colony counts, the number of spots representing effector T cells secreting IFN- $\gamma$ in an ELISPOT, and the levels of Th1, Th2, and Th17 cytokines, which were measured by a cytometric bead array (CBA). Mouse RNA samples were subjected to transcriptome sequencing.

Results: After 13 weeks of treatment, the mean histopathological lesion area of the NBXH group was significantly smaller than that of the TB model group $(P<0.05)$. Compared with those in the TB model group, the lung colony counts in the JHW and NBXH groups were significantly decreased $(P<0.05)$, and the IL-2 and IL-4 levels in the NBXH group were significantly increased $(P<0.05)$. NBXH partly restored significant changes in gene expression caused by Mycobacterium tuberculosis (M. tuberculosis) infection. According to GO and KEGG analyses, the changes in biological process (BP), cell composition (CC) and molecular function (MF) terms and in signaling pathways caused by NBXH and $\mathrm{JHW}$ treatment were not completely consistent, but they were mainly related to the immune response and inflammatory response in the mouse TB model.
\end{abstract}

Conclusions: NBXH had therapeutic effects similar to those of JHW in improving lung histopathology, reducing lung colony counts, and regulating the levels of cytokines. NBXH restored significant changes in gene expression and repaired cell damage caused by M. tuberculosis infection by regulating immune-related pathways, which clarified the mechanism of action of $\mathrm{NBXH}$.

Keywords: Traditional Chinese medicine, Tuberculosis, NiuBeiXiaoHe extract, JieHeWan, Therapeutic effect

\footnotetext{
*Correspondence: zhengy309@126.com; xueqiongwu@139.com

${ }^{+}$Li-Yao Duan and Yan Liang contributed equally to this work.

${ }^{1}$ Tuberculosis Prevention and Control Key Laboratory, Beijing Key Laboratory of New Techniques of Tuberculosis Diagnosis and Treatment, Tuberculosis Research Institute, the 8th Medical Center, Chinese PLA General Hospital, Beijing 100091, China

Full list of author information is available at the end of the article
}

(c) The Author(s). 2021 Open Access This article is licensed under a Creative Commons Attribution 4.0 International License, which permits use, sharing, adaptation, distribution and reproduction in any medium or format, as long as you give appropriate credit to the original author(s) and the source, provide a link to the Creative Commons licence, and indicate if changes were made. The images or other third party material in this article are included in the article's Creative Commons licence, unless indicated otherwise in a credit line to the material. If material is not included in the article's Creative Commons licence and your intended use is not permitted by statutory regulation or exceeds the permitted use, you will need to obtain permission directly from the copyright holder. To view a copy of this licence, visit http://creativecommons.org/licenses/by/4.0/. The Creative Commons Public Domain Dedication waiver (http://creativecommons.org/publicdomain/zero/1.0/) applies to the data made available in this article, unless otherwise stated in a credit line to the data. 


\section{Background}

Tuberculosis (TB) is a major social and public health problem worldwide. In 2019, there were 10 million new TB cases, and 1.2 million people died from TB worldwide [1]. In recent years, the rates of multidrug-resistant and extensively drug-resistant TB have been increasing. Anti-TB chemotherapeutic drugs have relatively high toxicity and side effects. The significant advantage of traditional Chinese medicine treatments is that they have a comprehensive therapeutic effect with "multicomponent, multitarget, and multisystem" features. Adjuvant treatment of multidrug-resistant tuberculosis (MDR-TB) with traditional Chinese medicine can effectively alleviate the symptoms of $\mathrm{TB}$, promote the repair of $\mathrm{TB}$ damage, and treat or prevent adverse reactions to chemotherapeutic drugs. In China, there are rich resources for Chinese herbal medicine, and the treatment of TB with traditional Chinese medicine has a long history, which has accumulated rich experience and developed many mature prescriptions and traditional Chinese medicine preparations, such as JieHeWan (JHW), BaiHeGuJinTang, and KangLaoWan.

The traditional Chinese medicine prescription NiuBeiXiaoHe (NBXH) has been used in the clinic for 16 years. It is composed of 6 traditional Chinese medicines: Bulbus Fritillariae Cirrhosae, Rhizoma Bletillae, Herba Houttuyniae, Radix Platycodonis, Fructus Arctii, and glutinous rice. Among these components, Bulbus Fritillariae Cirrhosae and Rhizoma Bletillae are used as the monarch herbs. Herba Houttuyniae, Radix Platycodonis, and Fructus Arctii are used as the minister herbs, and glutinous rice is used as the adjuvant and guide herb. Clinical research has shown that NBXH containing these 6 traditional Chinese medicines with reasonable compatibility had the effects of nourishing Yin, moistening the lungs, relieving cough, resolving phlegm, clearing heat, detoxification, and softening hard modes, which could eliminate TB symptoms, such as low fever, emaciation, cough, chest pain, and hemoptysis [2-4]. A previous study by Wang XM found that NBXH had a certain therapeutic effect on a mouse TB model [5]. Li GM [6] found that NBXH might play an anti-TB role by inhibiting the energy metabolism, protein synthesis, and antioxidative stress of M. tuberculosis, weakening the viability of $M$. tuberculosis in macrophages and affecting latent infection. Ling YB et al. [7] found that NBXH has an immunomodulatory effect through proteomics, this effect may down-regulate the JNK signaling pathway and inhibit the inflammatory response. Chen D [8] found that NBXH has no significant effects on the nervous system, immune function, or liver and kidney functions of normal mice and do not cause an acute toxicity reaction.

The Chinese medicine preparation JHW is made of 16 traditional Chinese medicines including tortoise shell processing with vinegar, oyster, turtle shell processing with vinegar, Rehmannia, prepared Rehmannia, amethyst (calcined), asparagus cochinchinensis, honey-fried Radix Stemonae, keel, Glehnia littoralis, donkey hide gelatin, Radix Ophiopogonis, cooked rhubarb, Rhizoma Bletillae, Bulbus Fritillariae Cirrhosae, and beeswax. JHW has the effects of nourishing Yin, moistening the lungs, nourishing the kidneys, strengthening body resistance, protecting against $\mathrm{TB}$ and killing $M$. tuberculosis. The results reported by Zhang JG et al. [9] showed that JHW combined with anti-TB chemotherapeutic drugs could improve the immune function of the body, enhance the sensitivity to chemotherapeutic drugs, and alleviate the side effects of chemotherapeutic drugs.

In this study, the traditional Chinese medicine preparation JHW, which is used in the clinic, was used as a positive control to compare the anti-TB effects of NBXH and JHW for the first time in vivo, to study the effect of $\mathrm{NBXH}$ on multiple cytokines and gene expression profiles in a mouse infection model, to clarify the anti-TB mechanism of $\mathrm{NBXH}$, to find a therapeutic effect evaluation index applicable to traditional Chinese medicine, and to provide a reliable experimental basis for the development of a new anti-TB traditional Chinese medicine preparation.

\section{Methods \\ Traditional Chinese medicines \\ NBXH was prepared by Xi'an XinTong Pharmaceutical Research Company, Ltd. (Xi'an, China). JHW was pro- duced by Anhui ZhiFeiLongKeMa Biopharmaceutical Company, Ltd. (Anhui, China).}

\section{Mice}

Specific pathogen-free female BALB/c mice, aged 6-8 weeks, 17-21 g, were obtained from the Vital River Laboratory Animal Technology Company, Ltd. (Beijing, China). They were maintained under barrier conditions in an animal room in the 8th Medical Center of the Chinese PLA General Hospital, Beijing, China. The experiments involving animals were approved and conducted by the Animal Ethical Committee of the 8th Medical Center of the Chinese PLA General Hospital, and mouse care met the standards of the Experimental Animal Regulation Ordinances defined by the China National Science and Technology Commission.

\section{M. tuberculosis strain}

The standard $M$. tuberculosis strain H37Rv was purchased from the Chinese Academy for Food and Drug Control (Beijing, China). Colony forming units (CFUs) were used to determine amounts of viable bacteria by plating serial dilutions on Lowenstein-Jensen medium. 


\section{Establishment of a mouse TB model and experimental grouping}

$\mathrm{BALB} / \mathrm{c}$ mice aged 6-8 weeks were injected intravenously via the tail vein with $3.1 \times 10^{5}$ CFUs of $M$. tuberculosis. At 3 days after infection, 3 mice were randomly selected and euthanized, and their lungs and livers were taken for bacterial colony counting and pathological examination of lung tissue to observe whether the mouse TB model was successfully established. In addition, the other mice were randomly grouped. In the TB model group, 20 mice were gavaged daily with 0.5 $\mathrm{mL}$ of distilled water. In the JHW group, 20 mice were treated with $300 \mathrm{mg}$ of JHW per kilogram of body weight $(6 \mathrm{mg} / 0.5 \mathrm{ml} /$ day). In the NBXH group, 20 mice were treated with $200 \mathrm{mg}$ of NBXH per kilogram of body weight $(4 \mathrm{mg} / 0.5 \mathrm{ml} /$ day). In the normal control group, 20 mice were not infected with $M$. tuberculosis and were kept in an animal laboratory.

\section{Lung histopathological examination}

The right lung of mice was fixed with $10 \%$ formalin buffer and embedded in paraffin. Sections were prepared by the paraffin-embedded tissues, stained with hematoxylin and eosin, and then analyzed by a registered pathologist.

\section{Bacterial counts}

The left lung and upper liver of mice were homogenized in saline. Tissue suspensions were serially diluted 10fold. In addition, $0.1 \mathrm{ml}$ of each dilution was plated in duplicate on Lowenstein-Jensen plates and incubated at $37^{\circ} \mathrm{C}$ for 4 weeks. M. tuberculosis colonies were enumerated on each plate, and the results are expressed as CFUs per organ.

\section{Enzyme-linked immunospot assay (ELISPOT)}

A splenocyte suspension $\left(3 \times 10^{6} / \mathrm{ml}\right)$ and an ELISPOT plate were prepared in advance. The suspension was plated at $100 \mu \mathrm{l} /$ well. One well contained $50 \mu \mathrm{l}$ of complete cell culture medium, one well contained $50 \mu \mathrm{l}$ of $60 \mu \mathrm{g} / \mathrm{ml} \mathrm{PHA}$, and three wells contained $50 \mu \mathrm{l}$ of $60 \mu \mathrm{g} / \mathrm{ml}$ recombinant CE protein. The ELISPOT plate was incubated in a $37^{\circ} \mathrm{C}$ cell incubator containing $5 \%$ $\mathrm{CO}_{2}$ for $24 \mathrm{~h}$.

\section{Cytometric bead array (CBA)}

A splenocyte suspension $\left(3 \times 10^{6} / \mathrm{ml}\right)$ was seeded in 48 well cell culture plates at $200 \mu \mathrm{l}$ per well. Then, $200 \mu \mathrm{l}$ of complete cell culture medium, $100 \mu \mathrm{l} 60 \mu \mathrm{g} / \mathrm{mL}$ PHA, or $100 \mu \mathrm{l} 60 \mu \mathrm{g} / \mathrm{ml}$ recombinant CE protein was added to each well. After incubation in a $\mathrm{CO}_{2}$ incubator at $37^{\circ} \mathrm{C}$ for $48 \mathrm{~h}$, the culture supernatant was collected. The levels of cytokines in the splenocyte culture supernatant were detected according to Th1/Th2/Th17 cytokine CBA kit (BD Company, America) instructions.

\section{PBMC isolation and total RNA extraction}

Blood samples were collected from mice in an ethylenediaminetetraacetic acid dipotassium (EDTA) anticoagulant tube, and peripheral blood mononuclear cells (PBMCs) were isolated with the Mouse PBMC Isolation Kit (Haoyang Biological Products Technology, Tianjin, China). Total RNA was extracted from PBMCs using TRIzol Reagent (Life Biotechnology, Shanghai, China) according to the manufacturer's instructions. The total RNA concentration and purity were determined using a NanoDrop 2000 (Thermo Fisher Scientific, DE, USA). RNA integrity was assessed using the RNA Nano 6000 Assay Kit for an Agilent Bioanalyzer 2100 system (Agilent Technologies, CA, USA).

\section{Transcriptome sequencing}

Mouse RNA samples were subjected to RNA-Seq library preparation and transcriptome sequencing at Beijing Biomarker Biotechnology Co., Ltd. (Beijing, China). The sequencing libraries were generated using the Illumina NovaSeq platform (NEB, USA). Briefly, mRNA was enriched from total RNA using oligo (dT) magnetic beads. The mRNA was fragmented using divalent cations under an elevated temperature in fragmentation buffer. Using the mRNA as a template, the first cDNA strand was synthesized using random hexamer primers, and then the second cDNA strand was synthesized with buffer, dNTPs, RNase $\mathrm{H}$, and DNA polymerase I. The generated double-stranded cDNA was purified with AMPure XP beads. Then, the purified double-stranded cDNA was subjected to end repair and poly(A) tail addition, and sequencing adaptors were ligated to the fragments. Fragments (240 bp) were purified with AMPure XP beads. The cDNA library was enriched by PCR. Finally, library quality assessment and quantification were performed on an Agilent Bioanalyzer 2100 system by Q-PCR, followed by sequencing on an Illumina NovaSeq platform.

\section{Data analyses}

The significantly differentially expressed (DE) genes between the TB model group and normal control group and between the JHW or NBXH group and the TB model group were identified by volcano plots and hierarchical clustering. DE gene analysis between two groups was performed using the DESeq $\mathrm{R}$ package (1.10.1). Gene ontology (GO) enrichment analysis and Kyoto Encyclopedia of Genes and Genomes (KEGG) pathway analysis of the DE genes were implemented with the clusterProfiler $\mathrm{R}$ package. 


\section{Statistical analyses}

Statistical analysis of data was carried out using SAS (version 9.1, SAS Institute Inc., Cary, NC, USA). All the data are expressed as the mean \pm standard deviation (SD). The significance of differences among groups was evaluated by one-way analysis of variance (ANOVA). A $P$-value less than 0.05 indicated statistical significance. Gene expression profiling of mice was analyzed by assessing fold changes. The threshold used to screen up- or down-regulated genes was |Fold Change| $>1$ and $P$-value $<0.05$.

\section{Results}

Histopathological changes

On the third day after infection, pathological examination of lung tissues from mice showed that the alveolar septum was congested, thickened, and infiltrated with inflammatory cells. After 3 weeks of treatment, lung tissue sections from mice in each group showed extensive pathological changes, destruction of the alveolar structure, congestion and thickening of the alveolar septum with lymphocyte infiltration, and multiple tuberculous nodules formed by lymphocyte aggregation (Fig. 1a).

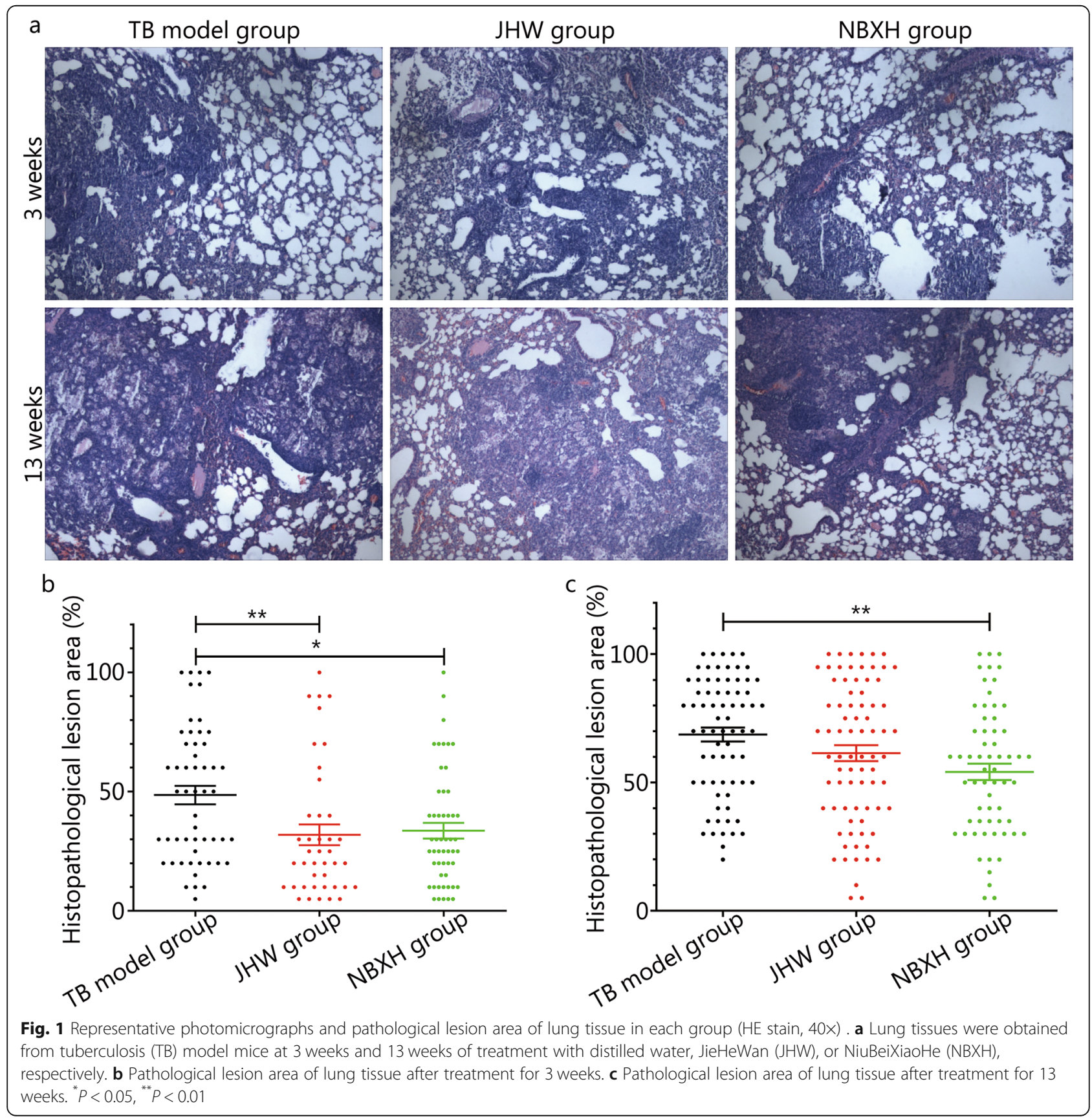


The pathological damage in the TB model group was more serious, with an average pathological damage area of more than $48.58 \%$. Compared with that in the TB model group, the pathological damage in the JHW group and NBXH group was significantly reduced $(P<0.05)$, additionally, the pathological area of lung tissue in both groups was decreased (31.95 and $33.65 \%$, respectively) (Fig. 1b). After 13 weeks of treatment, mouse lung tissue from each group showed severe pathological changes, including severe destruction of the alveolar structure and thickening and congestion of the alveolar septum, accompanied by a large amount of lymphocyte infiltration, and foam-like cells and lymphocytes aggregated to form tubercle nodules (Fig. 1a). The pathological damage in the TB model group was severe and extensive, and the average pathological lesion area was $68.68 \%$. The average pathological lesion area in the JHW group (61.41\%) was not significantly different from that in the TB model group $(P>0.05)$, that in the NBXH group $(54.11 \%)$ was significantly smaller than that in the TB model group $(P<0.05$, Fig. 1c) .

\section{Bacterial counts in the lungs and liver}

The lung colony counts of the TB model group at 3 days after infection were $\log _{10}(3.16 \pm 0.22)$, and the liver colony counts were $\log _{10}(4.30 \pm 0.29)$. After 3 weeks of treatment, compared with the TB model group, the JHW group and the NBXH group showed no significant differences in lung or liver colony counts $(P>0.05)$ (Fig. 2 left). After 13 weeks of treatment, the lung colony counts in the JHW group and the NBXH group were significantly lower than those in the TB model group $(P<0.05)$, and the lung colony counts in the NBXH group were lower than those in the JHW group (Fig. 2a right). Compared with the TB model group, the JHW group and the NBXH group showed no significant

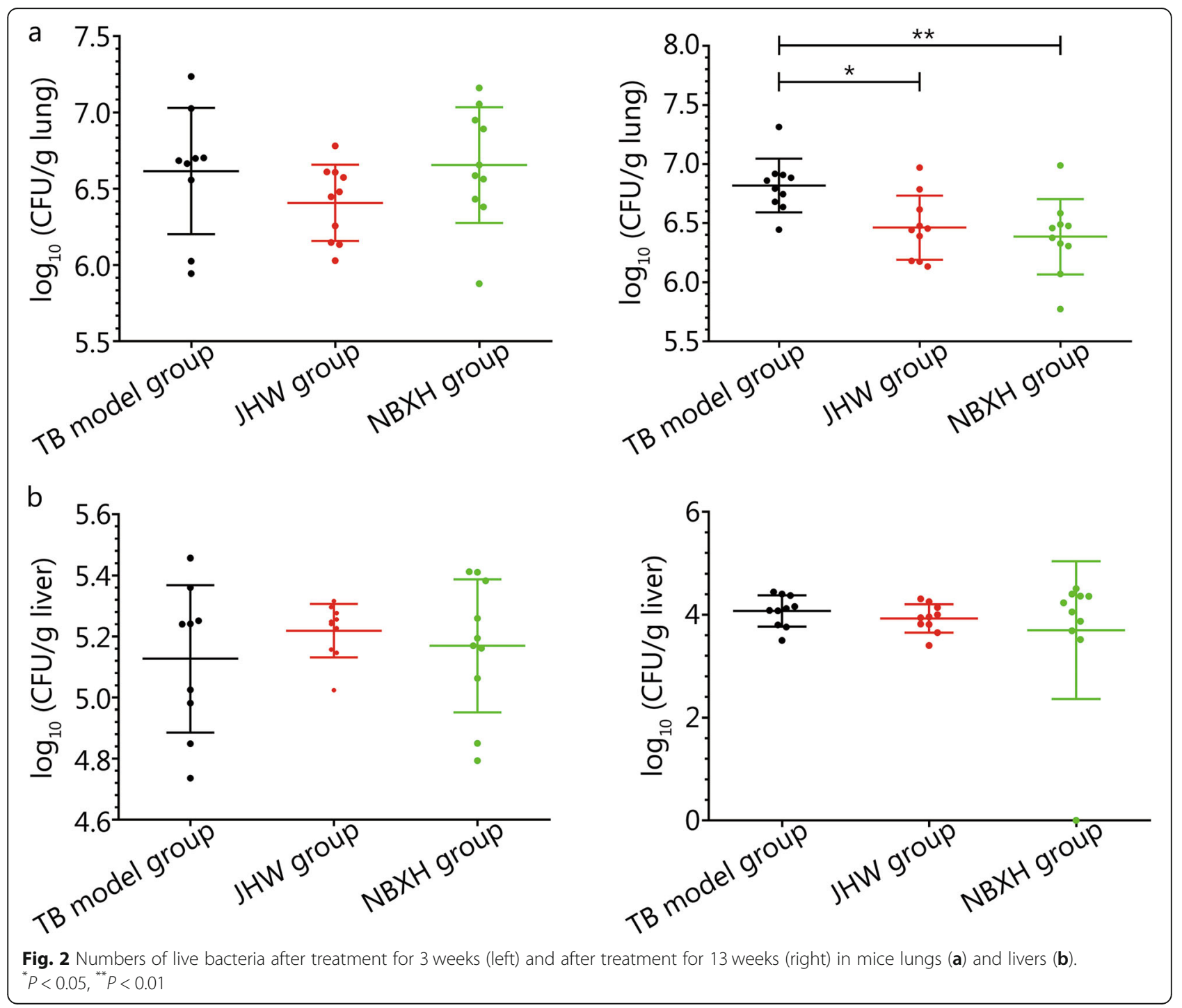


differences in liver colony counts $(P>0.05)$, but the liver colony counts in the NBXH group were relatively low (Fig. 2b right).

\section{Effector T cells secreting IFN- $\gamma$}

After 3 weeks of treatment, the numbers of spots representing effector $\mathrm{T}$ cells secreting IFN- $\gamma$ in the TB model group, JHW group, and NBXH group were all higher than that in the normal control group, but only the increase in the TB model group was statistically significant $(P<0.05)$. After 13 weeks of treatment, the numbers of spots for effector $\mathrm{T}$ cells secreting IFN- $\gamma$ in the $\mathrm{TB}$ model group, JHW group, and NBXH group were still higher than that in the normal control group, but only the increase in the JHW group was statistically significant, and that in the JHW group was significantly higher than that in the normal control group and NBXH group $(P<0.05)$. Compared with the TB model group, the JHW group and NBXH group did not show significant differences in the number of spots for effector T cells secreting IFN $-\gamma(P>0.05$, Fig. 3).

\section{Expression of 7 inflammatory cytokines}

After 3 weeks of treatment, compared with that in the normal control group, the IFN- $\gamma$ levels in the TB model group, JHW group, and NBXH group were significantly increased $(P<0.01)$, but there were no significant differences among the TB model group, JHW group, and NBXH group $(P>0.05)$. The IL-4 levels in the normal control group, JHW group, and NBXH group were significantly lower than that in the TB model group $(P<$ $0.001)$. The IL-17A level in the TB model group was significantly higher than that in the normal control group $(P<0.05)$, but there were no significant differences among the other groups $(P>0.05)$. There were no significant differences in IL-2, TNF, IL-6, or IL-10 levels $(P>0.05$, Fig. 4). After 13 weeks of treatment, the IFN- $\gamma$ levels of the TB model group and the JHW group were still significantly higher than that of the normal control group $(P<0.05)$. Compared with that of the TB model group, the IL-2 levels of the normal control group and NBXH group were significantly increased $(P<0.05)$. There was no significant difference in TNF levels among the groups $(P>0.05)$, but the TNF level in the NBXH group was highest. The IL-4 levels of the normal control group and NBXH group were significantly higher than those of the TB model group and JHW group $(P<0.05)$. There were no significant differences in IL-6, IL-10, or IL-17A levels among the groups $(P>0.05)$, but the IL-17A level was increased in the NBXH group (Fig. 5).

\section{Identification of DE genes before and after $M$. tuberculosis infection or JHW or NBXH treatment}

Heat map and volcano map are used to show the changes of gene expression profiles before and after $\mathrm{M}$. tuberculosis infection, JHW or NBXH treatment (Figs. S1, S2). The results showed that 2774 up-regulated and 2266 down-regulated DE genes were identified in the TB model group compared with the normal control group. A total of 148 up-regulated and 210 down-regulated DE genes were identified in the JHW group compared with the TB model group. A total of 220 up-regulated and 278 downregulated DE genes were identified in the NBXH group compared with the TB model group. Of the top 30 upregulated DE genes after NBXH treatment, 19 were down-regulated in the TB model group, and 11 were also up-regulated in the JHW group (shown in Table 1). Of the top 30 down-regulated DE genes after NBXH treatment, 23 were up-regulated in the TB model group, 11 were down-regulated in the JHW group, and 2 were upregulated in the JHW group (shown in Table 2).

\section{GO analyses of DE genes before and after $M$. tuberculosis infection or JHW or NBXH treatment}

The top 10 biological processes (BP) (Fig. 6), cellular components (CC) (Fig. 7), and molecular function (MF) (Fig. 8) GO terms with statistical significance for DE genes identified after $M$. tuberculosis infection or
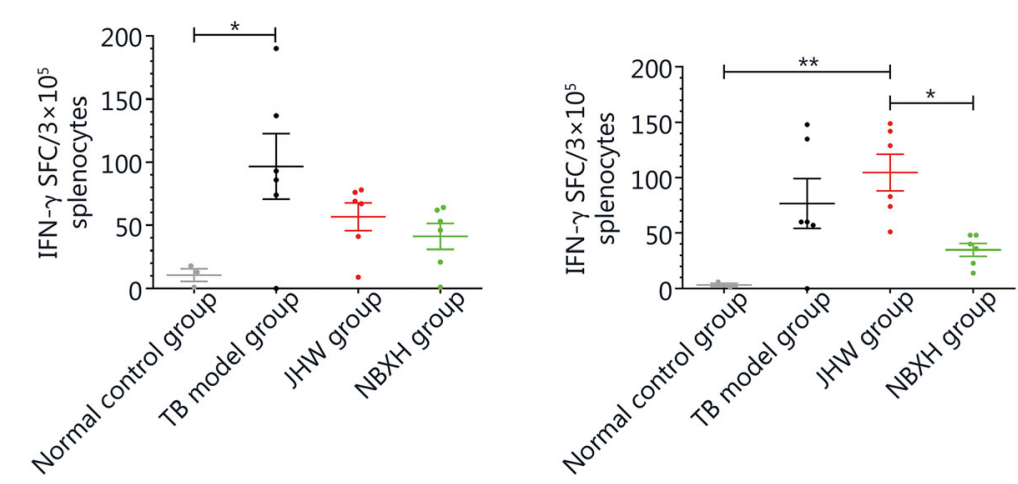

Fig. 3 ELISPOT analysis of splenocytes of mice in each group after treatment for 3 weeks (left) and 13 weeks (right). ${ }^{*} P<0.05,{ }^{* *} P<0.01$ 


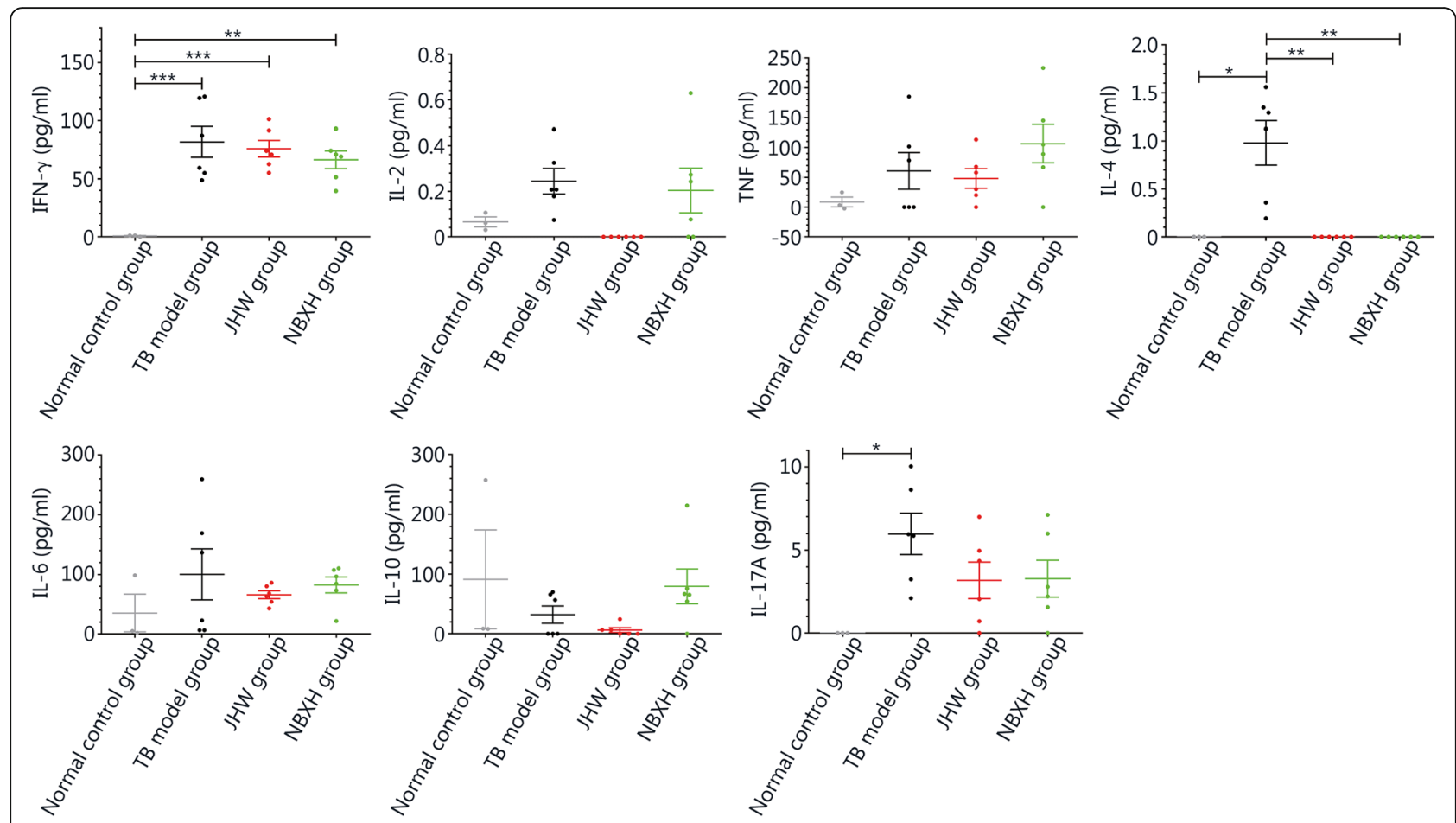

Fig. 4 Levels of cytokines in the spleen lymphocyte culture supernatants of mice in each group after treatment for 3 weeks. The cytokines detected were IFN- $\gamma$, IL-2, TNF, IL-4, IL-6, IL-10 and IL-17A, respectively. ${ }^{*} P<0.05,{ }^{* *} P<0.01,{ }^{* * *} P<0.001,{ }^{\#} P<0.0001$

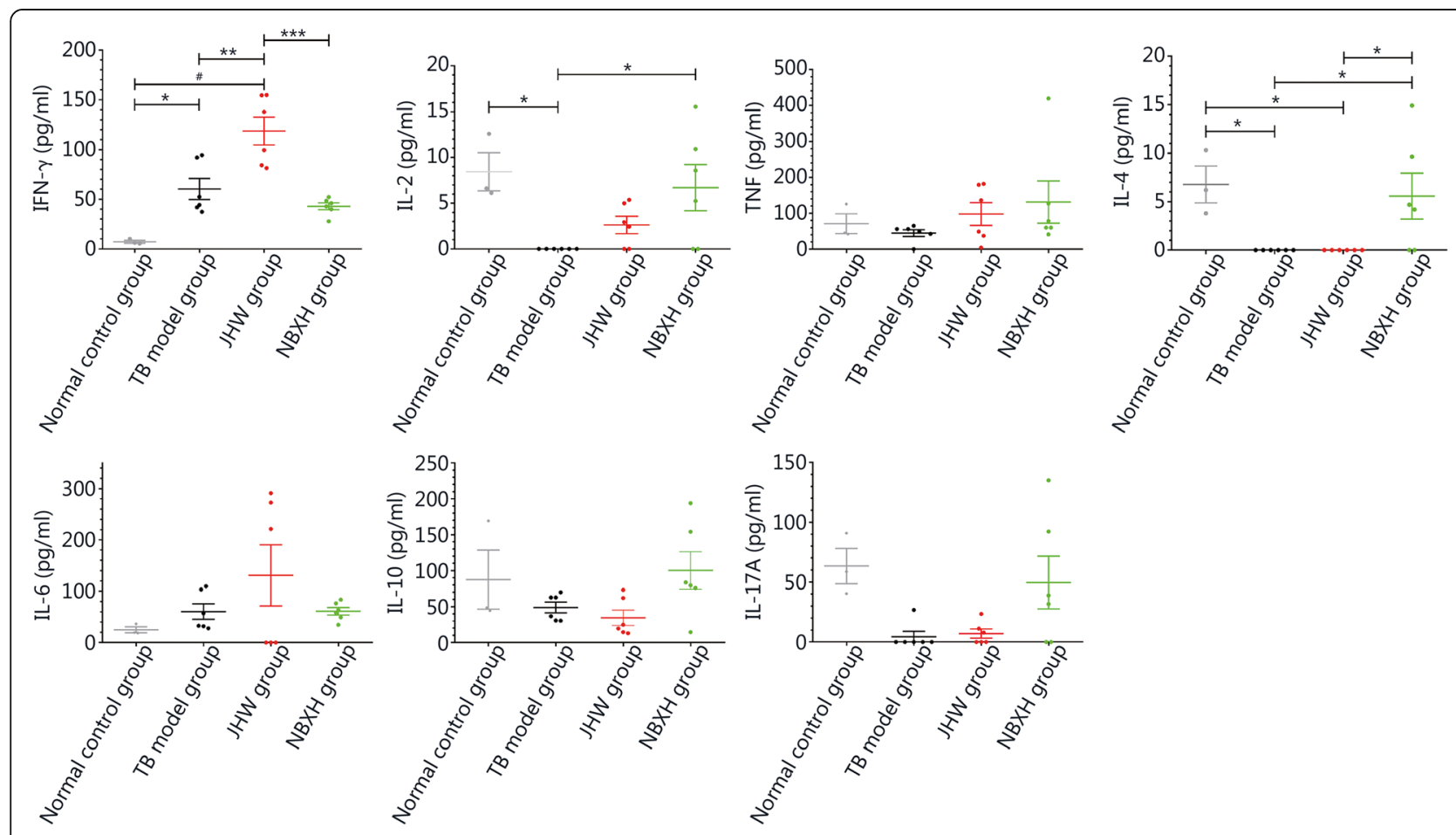

Fig. 5 Levels of cytokines in the spleen lymphocyte culture supernatants of mice in each group after treatment for 13 weeks. The cytokines detected were IFN- $\gamma$, IL-2, TNF, IL-4, IL-6, IL-10 and IL-17A, respectively. ${ }^{*} P<0.05,{ }^{* *} P<0.01,{ }^{* * *} P<0.001,{ }^{\#} P<0.0001$ 


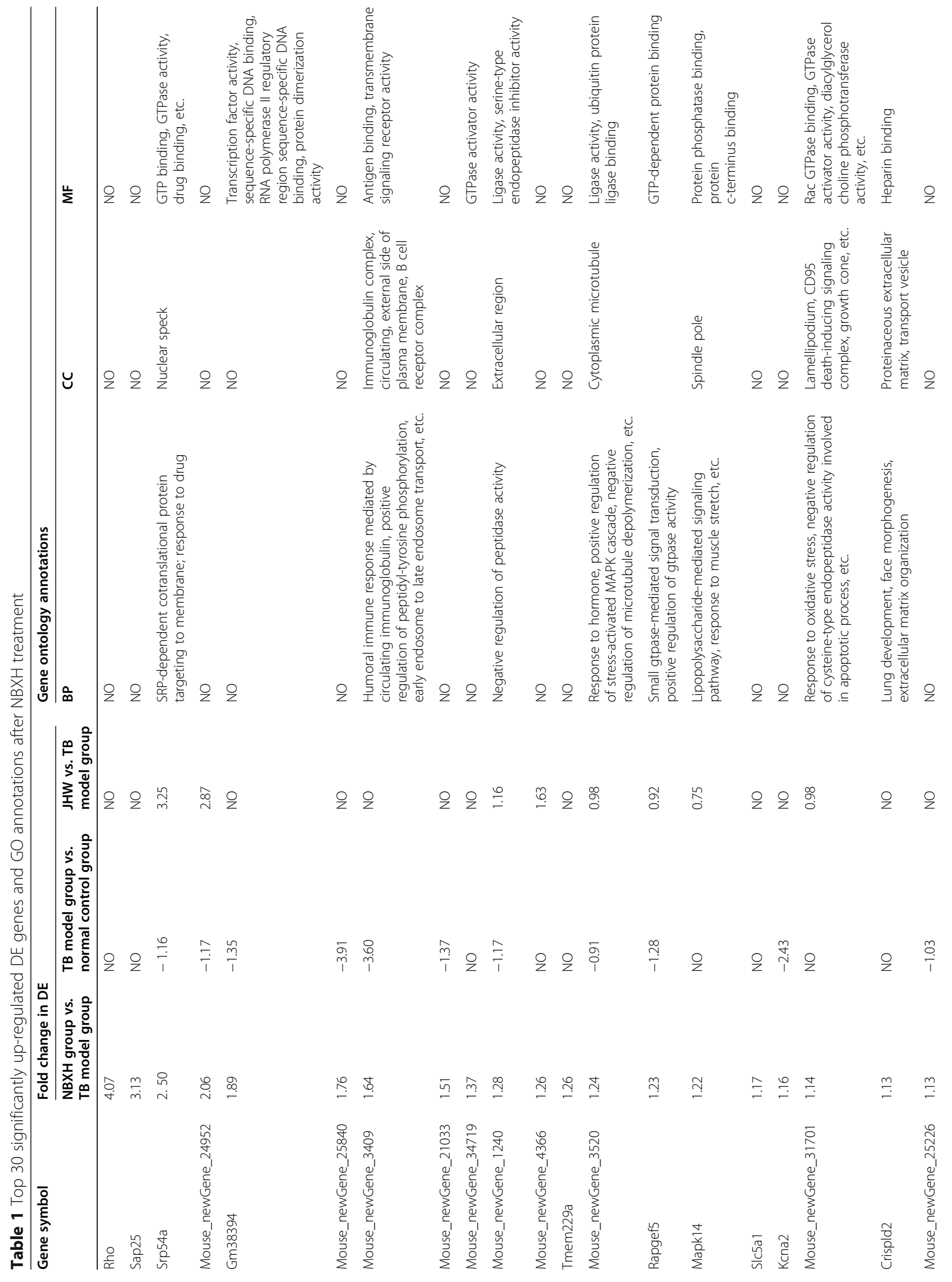




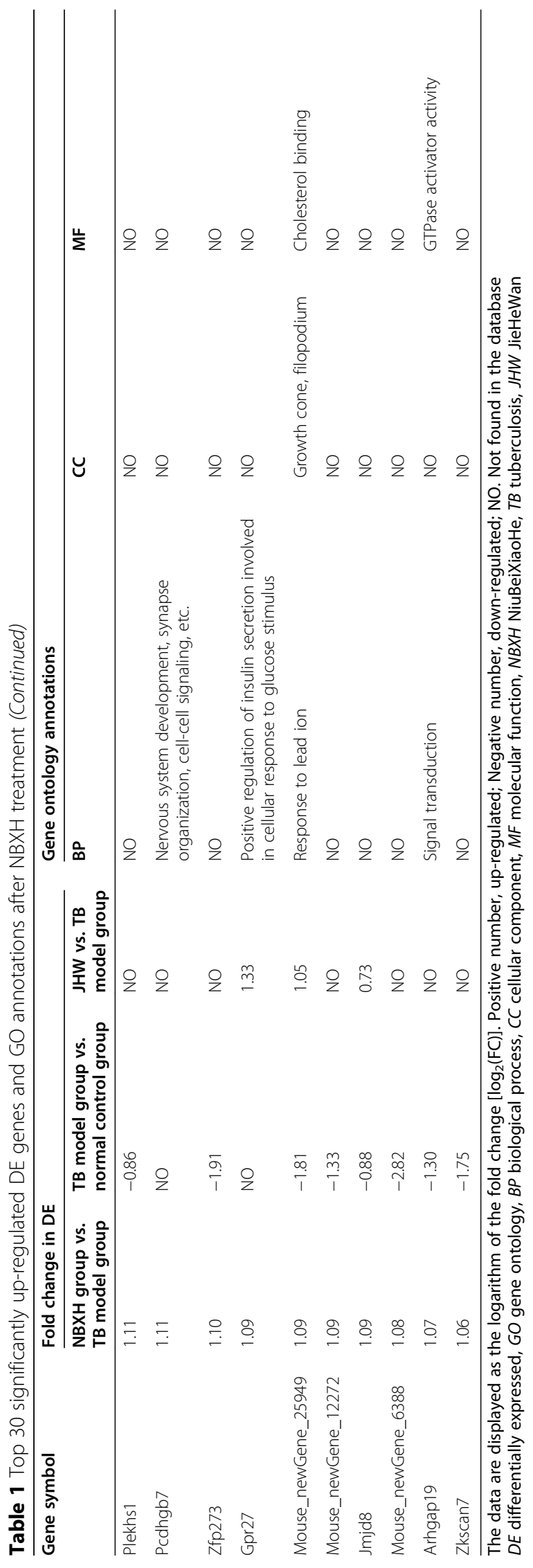




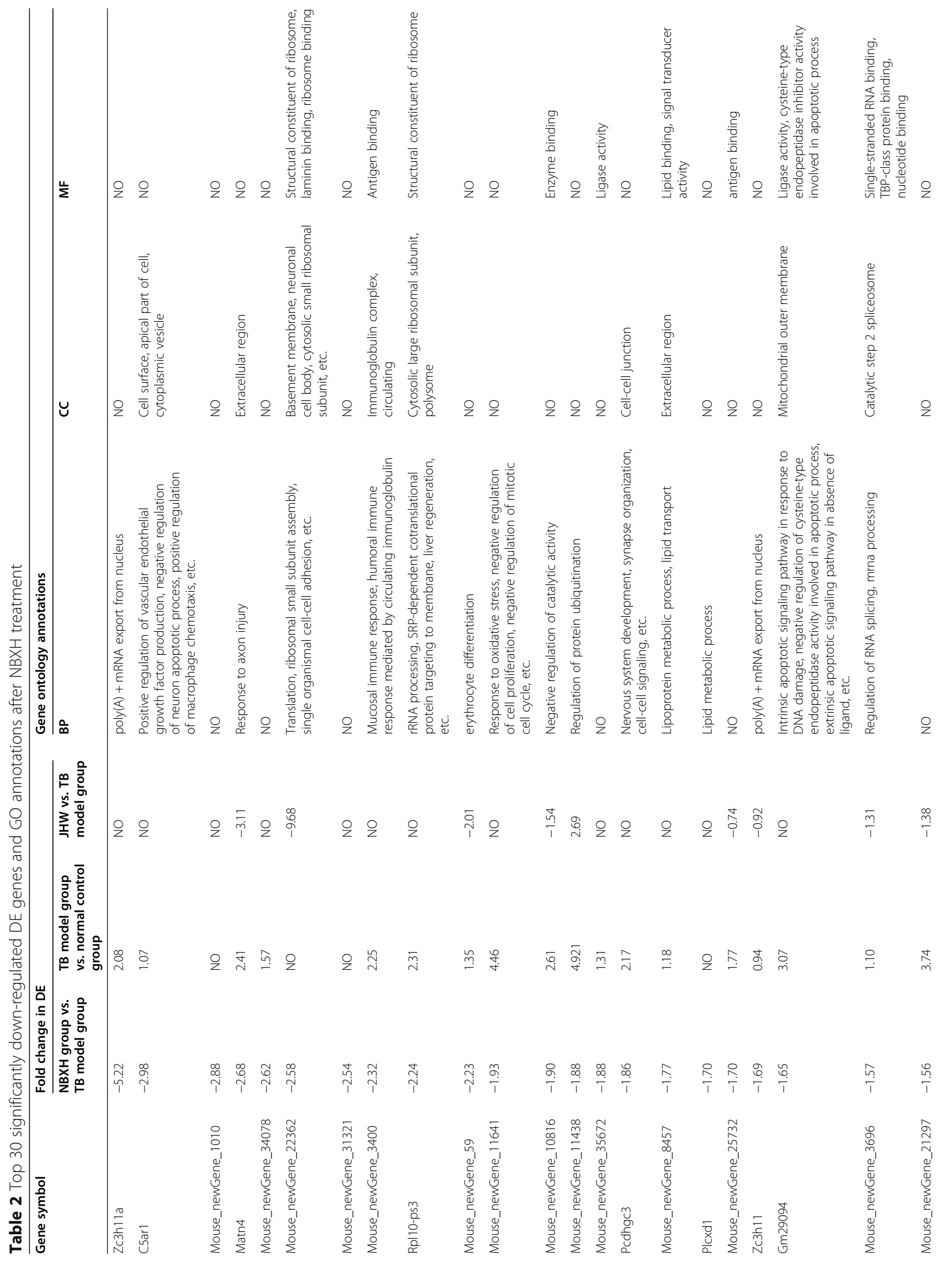


Dunn et al. Military Medical Research $\quad$ (2021) 8:34

Page 11 of 22

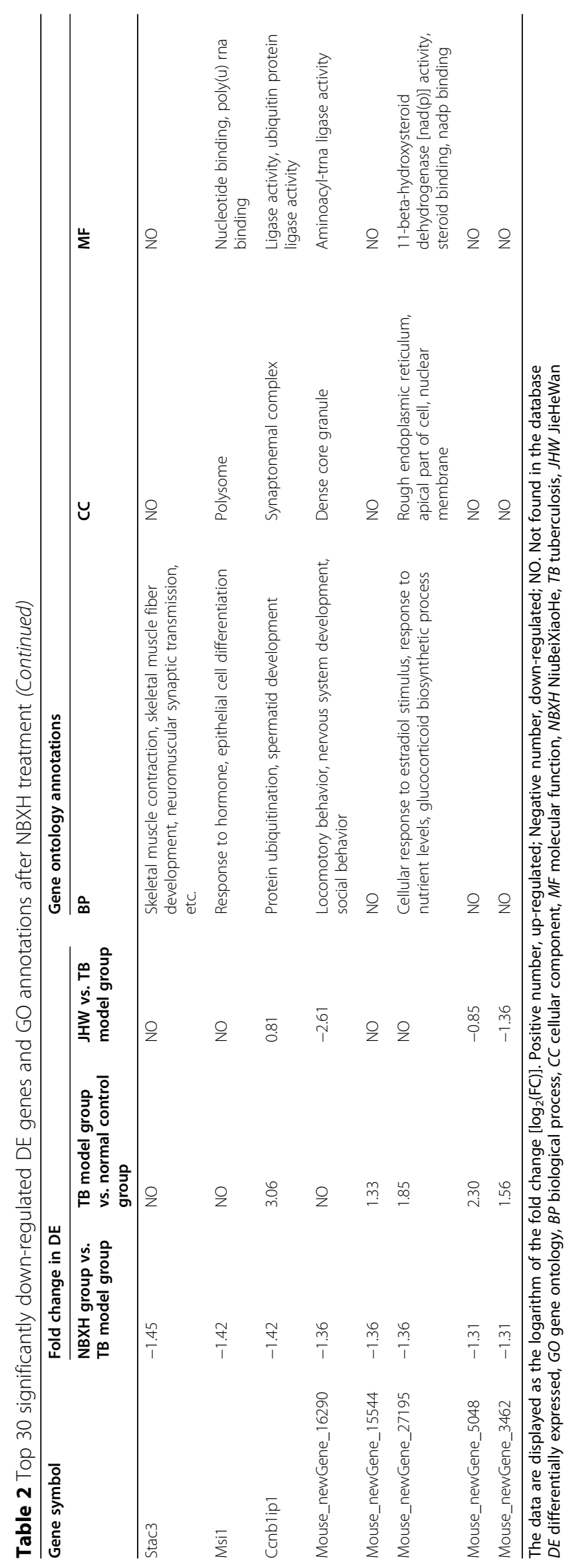


a

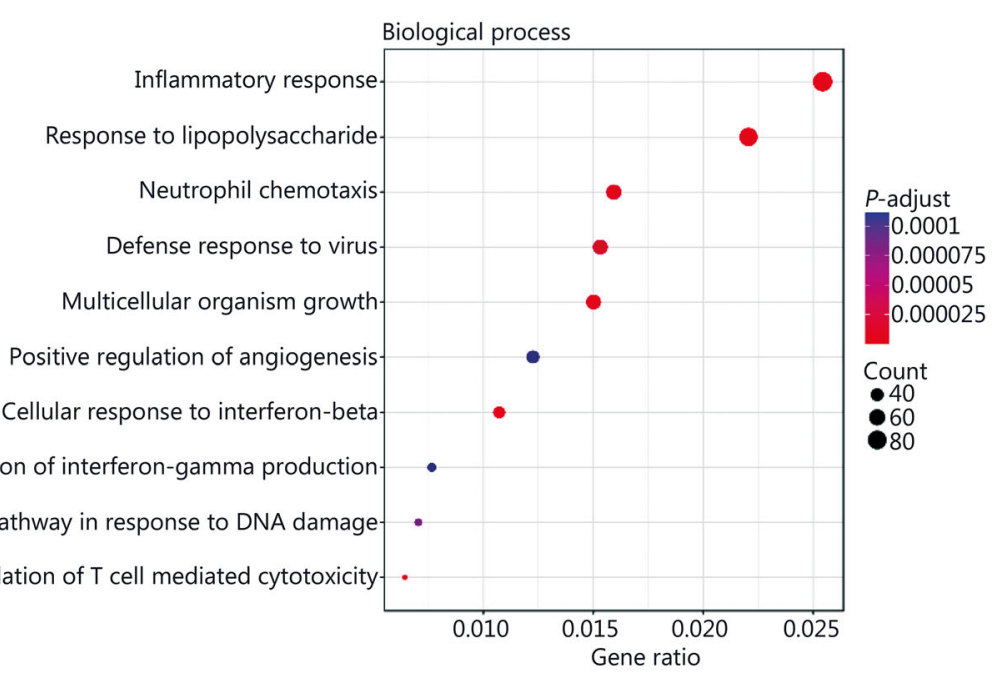

b

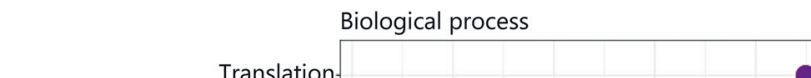
Intrinsic apoptotic signaling pathway in response to DNA damage

Positive regulation of $\mathrm{T}$ cell mediated cytotoxicity-

Biological process

Obsolete GTP catabolic process Negative regulation of I-kappaB kinase/NF-kappaB signaling Learning or memory B cell receptor signaling pathway Mitochondrial electron transport, $\mathrm{HADH}$ to ubiquinone Layer formation in cerebral cortex. I-kappaB phosphorylation- . RNA polymerase II transcriptional preinitiation complex assembly- .

Membrane protein intracellular domain proteolysis.

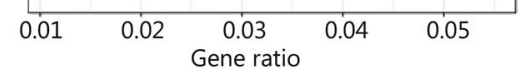

$$
\begin{aligned}
& \text { Count } \\
& : 2 \\
& : 4 \\
& 06 \\
& 8 \\
& 810 \\
& 10 \\
& P \text {-adjust } \\
& 0.3 \\
& 0.2 \\
& 0.1
\end{aligned}
$$

\section{C} Biological process

Negative regulation of cysteine-type endopeptidase activity. involved in apoptotic process Intrinsic apoptotic signaling pathway in response to DNA damage Extrinsic apoptotic signaling pathway in absence of ligand Lipopolysaccharide-mediated signaling pathwayDendrite developmentPositive regulation of vascular endothelial growth factor productionVesicle transport along microtubule. Regulation of I-kappaB kinase/NF-kappaB signaling. . Regulation of protein ubiquitination- .

Fig. 6 (See legend on next page.)

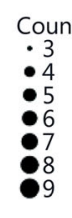
P-adjust

0.07855401 
(See figure on previous page.)

Fig. 6 Gene ontology (GO) analyses of significant differentially expressed (DE) genes identified by comparing before and after M. tuberculosis infection or NiuBeiXiaoHe (NBXH) treatment in biological process. The abscissa is the gene ratio, which is the ratio of the gene of interest annotated in the term to the number of all DE genes, and the ordinate is every GO term. Dot size represents the number of DE genes annotated in the pathway, and dot color represents the corrected $P$-value of the hypergeometric test. a Tuberculosis (TB) model group vs. normal control group. b JieHeWan (JHW) vs. TB model group. c NBXH vs. TB model group.

traditional Chinese medicines treatment are shown in Figs. 6, 7 and 8.

\section{Pathway analyses of DE genes before and after $M$. tuberculosis infection or JHW or NBXH treatment}

The molecular pathways of the identified DE genes were annotated by KEGG analysis. The results for the top 20 pathways closely related to the disease pathogenesis are shown in Fig. 9.

\section{Discussion}

The treatment of TB with a combination of traditional Chinese medicine and chemotherapeutic drugs can achieve a comprehensive therapeutic effect, reduce the symptoms of TB, reduce the liver and kidney damage induced by chemotherapeutic drugs, reduce complications, and improve the treatment effect in TB patients [10]. According to the report by Yang QS et al. [11], JHW has anti-M. tuberculosis activity, can enhance host immune function and has a certain therapeutic effect on many kinds of $\mathrm{TB}$, such as treatment-naive and previously treated pulmonary $\mathrm{TB}$, drug-resistant $\mathrm{TB}$, and senile $\mathrm{TB}$. Comparing the anti-TB effects of NBXH and JHW in vivo will provide a reliable theoretical basis for the clinical application of the anti-TB Chinese medicine compound NBXH.

The results of previous studies by our team showed that NBXH has a distinct degree of therapeutic effect on the mouse TB model, improving the general condition, reducing organ colony counts, and decreasing the severity of TB lesions [12]. To better understand the difference in the anti-TB effect between NBXH and traditional Chinese medicine in clinical application, this study compared the anti-TB effect of an NBXH powder newly produced by Xi'an XinTong Pharmaceutical Research Co., Ltd. and that of JHW in vivo. In this study, the TB model was confirmed to be successfully established by observing the lung tissue pathology and organ colony counts of mice infected with $M$. tuberculosis for 3 days.

The pathogenic mechanism of $M$. tuberculosis infection mainly involves the growth and metabolism of $M$. tuberculosis inducing the host immune system to produce an inflammatory response and cause immune damage. After M. tuberculosis infects the body, different types of pathological changes, such as exudation, hyperplasia, and necrosis, may occur depending on the number of infectious bacteria, bacterial virulence, host immune response, and tissue characteristics [13, 14].
The results published by Xiang ZG et al. [15] showed that 1 week after acute $M$. tuberculosis infection in mice, inflammatory cell exudation and interstitial edema appeared in the lungs, and inflammatory cell aggregation occurred in the spleen, 3 weeks later, granulomas appeared in the lungs and spleen, and the area and quantity of the inflammatory reaction increased with the extension of infection time. The application of HuangKuiSu could significantly reduce the pathological damage in lung tissue in mice infected with $M$. tuberculosis and reduce lung colony counts [16]. The application of FeiLaoKang could also significantly inhibit the inflammatory response in the lungs in a mouse TB model and significantly improved inflammatory symptoms [17]. The results of this study are consistent with these literature reports. The pathological examination of lung tissue in this study showed that the pathological changes in the NBXH group and JHW group were significantly less severe than those in the TB model group after 3 weeks of treatment, and the pathological area in the NBXH group was smaller than those in the TB model group and JHW group after 13 weeks of treatment. This shows that NBXH induces a better anti-TB inflammatory response and reduces pathological damage.

Organ bacterial loads are used to evaluate the antibacterial or bactericidal effect of anti-TB drugs in vivo. Organ bacterial loads correspond to the infection dose and last for at least 14 weeks [18]. The lower the organ bacterial loads are, the stronger the bactericidal effect of drugs, and the better the therapeutic effect [19]. Jiang JQ et al. showed that traditional Chinese medicine also had a certain bacteriostatic or bactericidal effect on M. tuberculosis, such as high-dose JHW significantly reducing lung and liver colony counts in guinea pigs (compared with the blank control group, the treated group showed a reduction of 272 CFUs $/ 10 \mathrm{mg}$ in the lungs and a reduction of 181 CFUs/10 $\mathrm{mg}$ in the liver), and reduces lung tissue lesions [20]. Our study showed that there were no significant differences in the bacterial loads of the lungs or liver in each group after 3 weeks of treatment, indicating that the treatment time of traditional Chinese medicines were too short to have a significant bacteriostatic or bactericidal effect. In contrast, after 13 weeks of treatment, the bacterial loads of the lungs in the NBXH group and JHW group were significantly lower than those in the TB model group, indicating that long-term traditional Chinese medicine treatment may 
a Cellular component

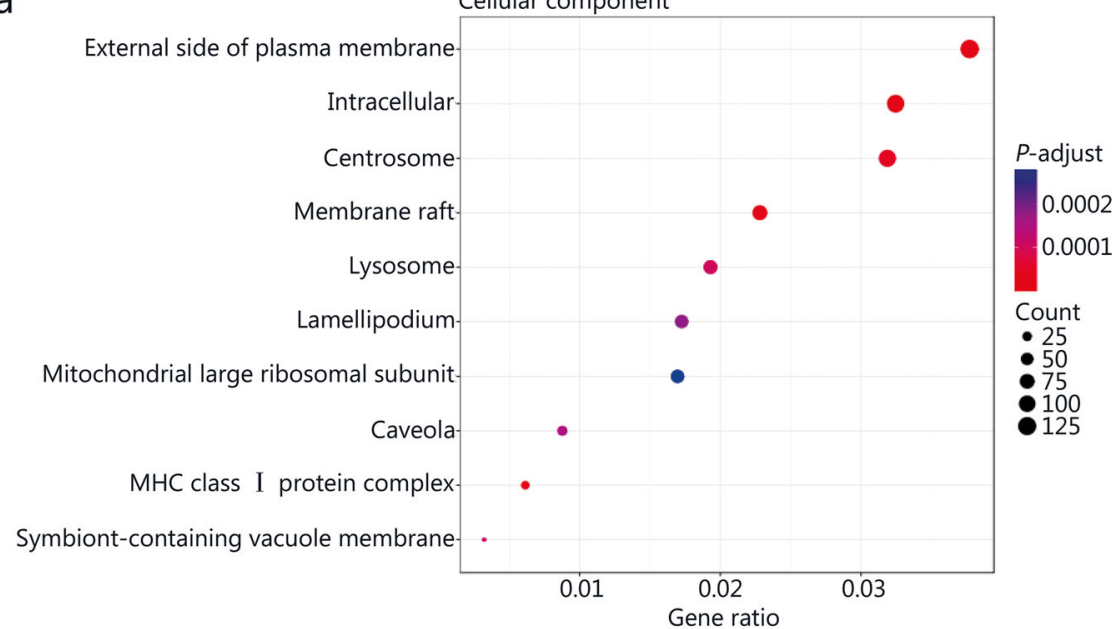

b

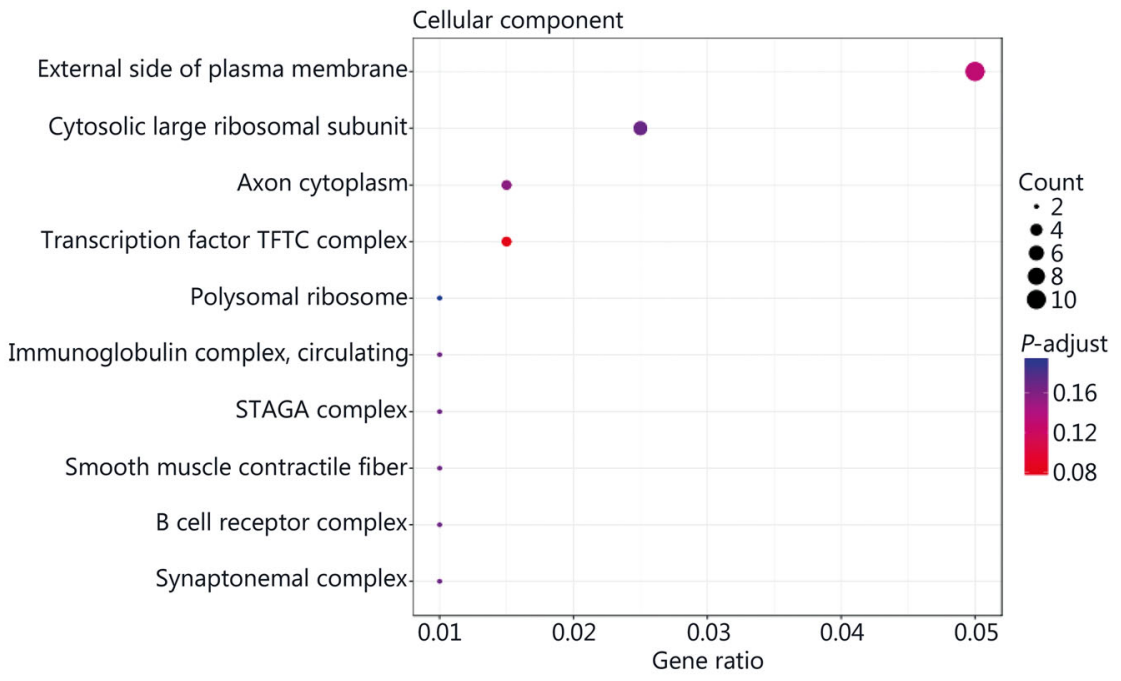

C
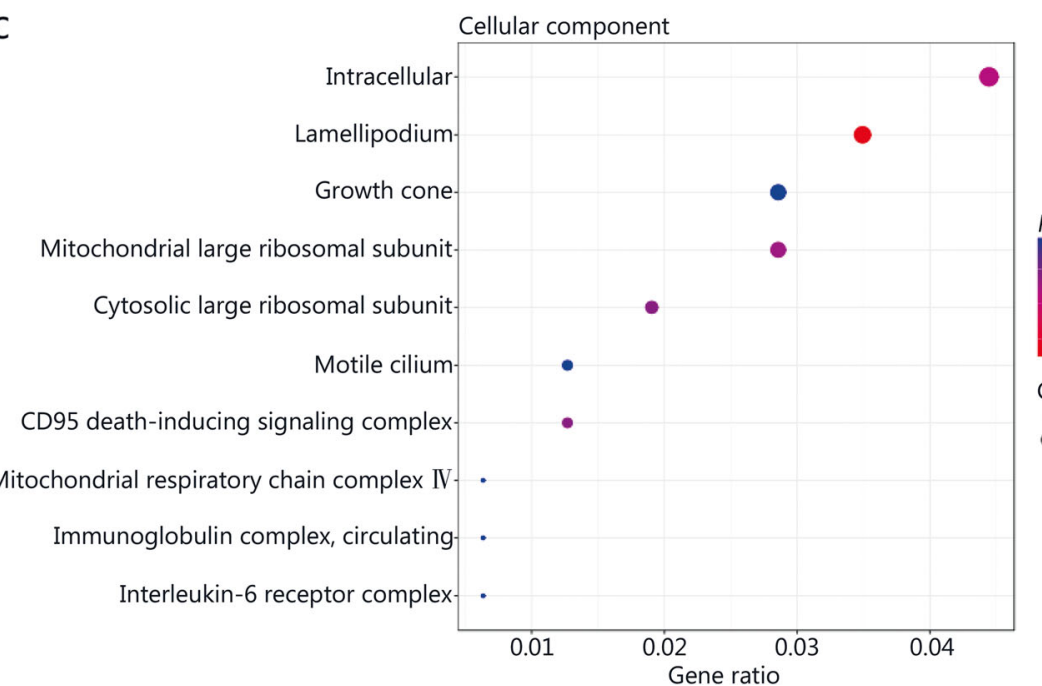
(See figure on previous page.)

Fig. 7 Gene ontology (GO) analyses of significant differentially expressed (DE) genes identified by comparing before and after M. tuberculosis infection or NiuBeiXiaoHe (NBXH) treatment in cellular component. The abscissa is the gene ratio, which is the ratio of the gene of interest annotated in the term to the number of all DE genes, and the ordinate is every GO term. Dot size represents the number of DE genes annotated in the pathway, and dot color represents the corrected $P$-value of the hypergeometric test. a Tuberculosis (TB) model group vs. normal control group. b JieHeWan (JHW) vs. TB model group. c NBXH vs. TB model group.

have better bacteriostatic and bactericidal effects on the lungs.

Traditional Chinese medicine can exert an anti-TB effect by regulating the host immune system. Helper $\mathrm{T}$ lymphocyte subsets and cytokines secreted by these cells play an important role in the process of anti-TB immunity. The Th1-type immune response is key in the anti- $M$. tuberculosis infection response. IFN- $\gamma$, IL-2, and TNF are the key immune indexes in the Th1-type immune response. The expression of IFN- $\gamma$ plays an important role in the anti-TB immune response, and the expression level in the body is closely related to TB [21-23]. IFN- $\gamma$ can promote the proliferation and differentiation of $\mathrm{T}$ cells, activate macrophages, and enhance the phagocytic activity and killing effect of $\mathrm{T}$ cells and macrophages on M. tuberculosis. IL-2 is an important factor in regulating the immune response, promoting the production of IFN- $\gamma$, and inducing the differentiation of killer cells, such as natural killer cells. TNF can cooperate with IFN$\gamma$ to enhance the expression of inducible nitric oxide synthetase and the antibacterial effect of macrophages and promote the formation of tuberculous granuloma [24, 25]. After infection with M. tuberculosis, the expression level of IFN- $\gamma$ generally increases, and after 2 months of intensive anti-TB treatment, the level of IFN- $\gamma$ decreases in responsive patients [26]. When the secretion of IL-2 and TNF is insufficient, the immune response cannot completely eliminate $M$. tuberculosis but is increased with the improvement in TB after antiTB treatment [27]. The cytokines secreted by Th2 cells are mainly IL-4, IL-6, and IL-10, and their main functions are to promote the development of B cells and induce a humoral immune response. IL-4 mainly promotes the differentiation of Th0 cells into Th2 cells and inhibits the expression of cytokines related to Th1 cells [28]. IL-6 is a B cell stimulator that can induce B cells to undergo differentiation and maturation to produce antibodies and participate in the anti-inflammatory effect of the body, at the same time, IL-6 can cooperate with colony-stimulating factor (CSF) to promote the growth and differentiation of primitive bone marrowderived cells and enhance the lytic function of natural killer (NK) cells. Liu WS et al. showed that the higher the level of IL-6 is in vivo, the more severe the lung inflammation [29]. IL-10 is an anti-inflammatory cytokine that can reduce the overactivation of coactivators in the phagocyte system to weaken antigen presentation [30]. It has been reported that the long-term control of TB infection requires not only the enhancement of the Th1type immune response but also the inhibition of the Th2-type immune response [31]. Lv Y et al. [32] showed that the mice infected with attenuated $M$. tuberculosis strain H37Ra mainly produced a Th1-type immune response. IL-17A is an inflammatory factor mainly produced by activated $\mathrm{T}$ cells that is induced in the early stage of $M$. tuberculosis infection and can promote the activation of $\mathrm{T}$ cells, participate in the recruitment of neutrophils and induce various cytokines, such as IL-6, IL-8, and granulocyte-macrophage colony-stimulating factor (GM-CSF) [33-35]. In addition, IL-17A participates in the formation of mature granuloma in the lungs during $M$. tuberculosis infection, which plays a key role in the prevention of M. tuberculosis spread [36]. In TB patients, Th1-type cytokines provide protective immunity, and Th2-type cytokines may promote the inflammatory response and immune damage, while Th17 cytokines play roles in both protection and pathological damage, regulating the $\mathrm{T}$ cell response is essential for promoting anti-M. tuberculosis immunity and preventing widespread immune pathology [37, 38]. In this study, results for the number of spots representing effector $\mathrm{T}$ cells secreting IFN- $\gamma$ and the levels of cytokines in the culture supernatant of splenic lymphocytes showed that after 3 weeks of treatment, the IFN- $\gamma$ levels in the TB model and two Chinese medicine treatment groups were significantly increased and the IL-4 level in the TB model group was also significantly increased, while the level of Th2-type cytokines did not increase in the two traditional Chinese medicine treatment groups, which suggested that Th1 and Th2 immune response were both enhanced in the TB model group, while the Th1 immune response was dominant in the two traditional Chinese medicine treatment groups. After 13 weeks of treatment, the IFN- $\gamma$ level in the TB model group and especially in the JHW group was still significantly increased, but the Th2 cytokine levels were not significantly increased, which indicated that the Th1 immune response was the main response and the Th2 immune response was inhibited in these two groups. The cytokine profile of the NBXH group changed, the IFN- $\gamma$ level decreased, the IL-2 level significantly increased, and the IL-4 and IL-17A levels increased significantly, which suggested that the Th1 immune response and Th2 immune response tended to be balanced. To date, studies 
a

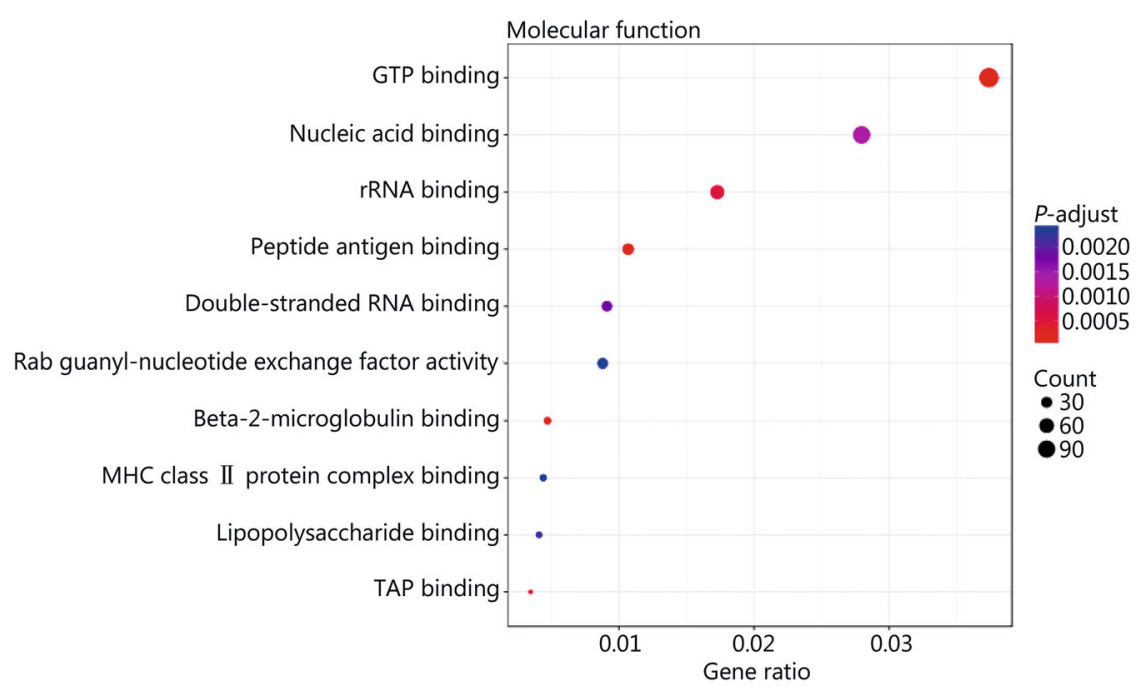

b

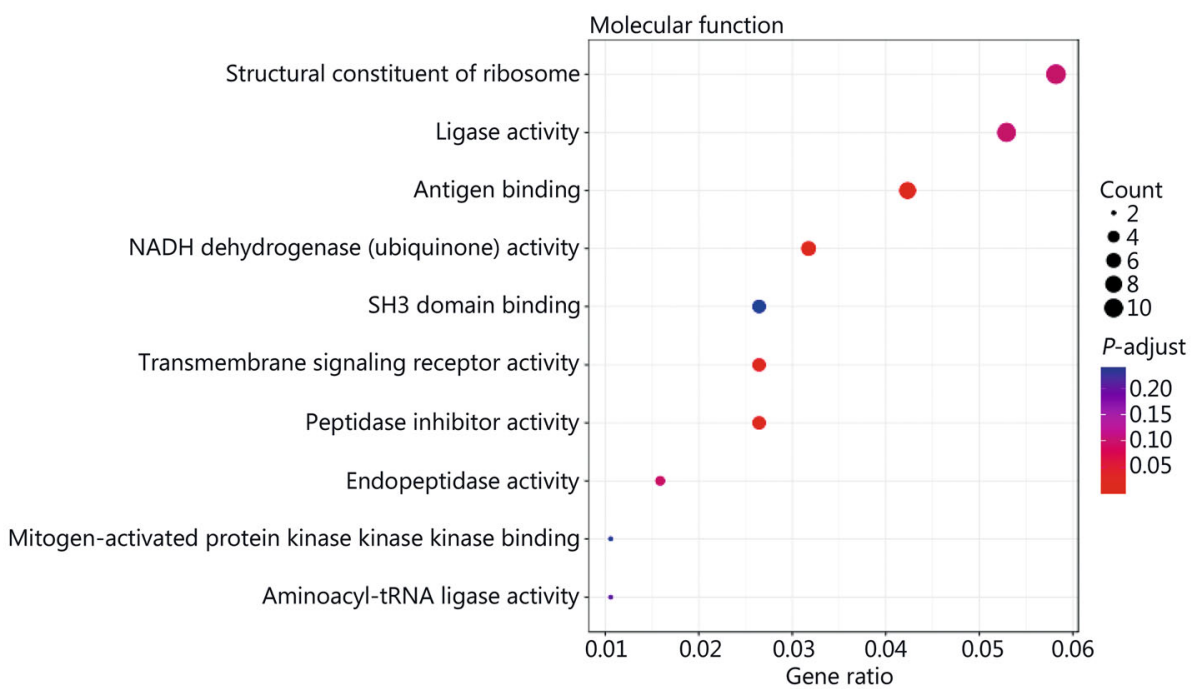

C

Transcription factor activity, sequence-specific DNA binding

Molecular function

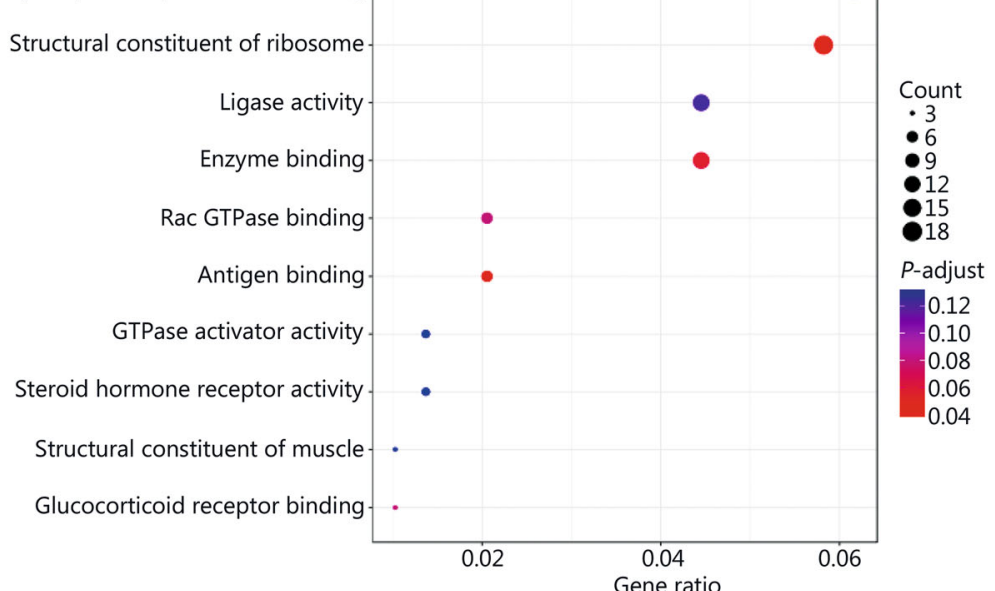

Fig. 8 (See legend on next page.) 
(See figure on previous page.)

Fig. 8 Gene ontology (GO) analyses of significant differentially expressed (DE) genes identified by comparing before and after M. tuberculosis infection or NiuBeiXiaoHe (NBXH) treatment in molecular function. The abscissa is the gene ratio, which is the ratio of the gene of interest annotated in the term to the number of all DE genes, and the ordinate is every GO term. Dot size represents the number of DE genes annotated in the pathway, and dot color represents the corrected $P$-value of the hypergeometric test. a Tuberculosis (TB) model group vs. normal control group. b JieHeWan (JHW) vs. TB model group. c NBXH vs. TB model group

have shown that in TB patients given effective anti-TB treatment, IFN- $\gamma$ levels decrease while IL-2 levels increase [26, 27], and these changes in the cytokine profile suggest effective NBXH treatment. In addition, some reports $[39,40]$ have shown that the recovery of Th1 and Th2 immune response balance in the treatment of TB is beneficial for the prognosis of patients. NBXH may play a role by regulating the Th1/Th2 immune balance rather than simply enhancing the Th1 response or weakening the Th2 response. After 13 weeks of treatment, the increase in the IL-17A level in some mice was conducive to the formation of granulomas and limited the spread of $M$. tuberculosis, which may also be one of the reasons for the reduced pathological damage in the $\mathrm{NBXH}$ group.

By comparing the changes in the gene expression profile before and after treatment with traditional Chinese medicine, we can understand the target of traditional Chinese medicine, clarify the mechanism of traditional Chinese medicine, and establish a new way to study the modernization of traditional Chinese medicine. At present, some research reports [41] have performed beneficial explorations, but we are the first to use transcriptome sequencing technology to analyze the abundance of mouse cell gene transcripts to evaluate the effect of anti-TB Chinese medicine treatment and explore the molecular mechanism of the effect. This study indirectly reflected the difference in gene expression by comparing the difference in gene transcript abundance between the TB model group and the normal control group and found 2774 up-regulated and 2266 downregulated $\mathrm{DE}$ genes, which is consistent with previous research reports $[42,43]$, which found that mycobacterial infection can cause significant changes in the gene expression profile of macrophages. Through GO and KEGG analyses, it was found that the cell damage that occurred after $M$. tuberculosis infection was relatively extensive, showing significant changes in terms for cell components, molecular functions and biological processes, the enriched GO terms related to BP were mainly inflammatory response, response to lipopolysaccharide, neutrophil chemotaxis, cellular response to interferonbeta, positive regulation of interferon-gamma production, intrinsic apoptotic signaling pathway in response, and positive regulation of $\mathrm{T}$ cell-mediated cytotoxicity, indicating that $M$. tuberculosis infection has a significant effect on the host immune response and inflammatory response. KEGG pathway analysis also shown that the pathways involved in antigen recognition, phagocytosis, apoptosis, antigen processing, and presentation by innate immune cells to adaptive immune cells all showed changes in up- or down-regulated pathways, such as the NOD-like receptor signaling pathway, phagosome, apoptosis, antigen processing and presentation, and cytokinecytokine receptor interaction. On the one hand, these findings support the current understanding of the pathogenesis of TB, that is, TB may be caused by inflammation and immune damage caused by $M$. tuberculosis proliferation in tissue cells [44]. On the other hand, they are helpful to further clarify the pathogenic mechanism of $M$. tuberculosis.

In this study, 148 up-regulated and 210 downregulated $\mathrm{DE}$ genes were identified by comparing the JHW group and the TB model group, and 220 upregulated and 278 down-regulated $\mathrm{DE}$ genes were identified by comparing the NBXH group with the TB model group. These results showed that the number of $\mathrm{DE}$ genes after NBXH treatment was slightly higher than that after JHW treatment, which reflects that NBXH has more targets than JHW, and traditional Chinese medicine compound preparations with complex chemical components have multicomponent, multipathway and multitarget therapeutic characteristics. The changes in $\mathrm{BP}, \mathrm{CC}$, and MF terms and in signaling pathways caused by treatment with $\mathrm{NBXH}$ or JHW were not completely consistent. NBXH treatment had a greater effect on BP terms and signaling pathways related to the immune response and inflammatory response, such as GO terms related to the biological processes response to oxidative stress, intrinsic apoptotic signaling pathway in response, lipopolysaccharide-mediated signaling pathway, regulation of l-kappaB kinase/NF-kappaB signaling, and regulation of protein ubiquitination. KEGG pathway analysis related to signaling pathways included down-regulated oxidative phosphorylation, up- or down-regulated Rap1 signaling pathway, and cytokine-cytokine receptor interaction. Among the pathways closely related to disease, the TB pathway ranked fifth. After JHW treatment, the pathways related to the regulation of the immune and inflammatory response were down-regulated Fc epsilon RI signaling pathway, protein processing in the endoplasmic reticulum and ribosome, up- or down-regulated TNF signaling pathway and notch signaling pathway, and down-regulated oxidative phosphorylation. These 
a

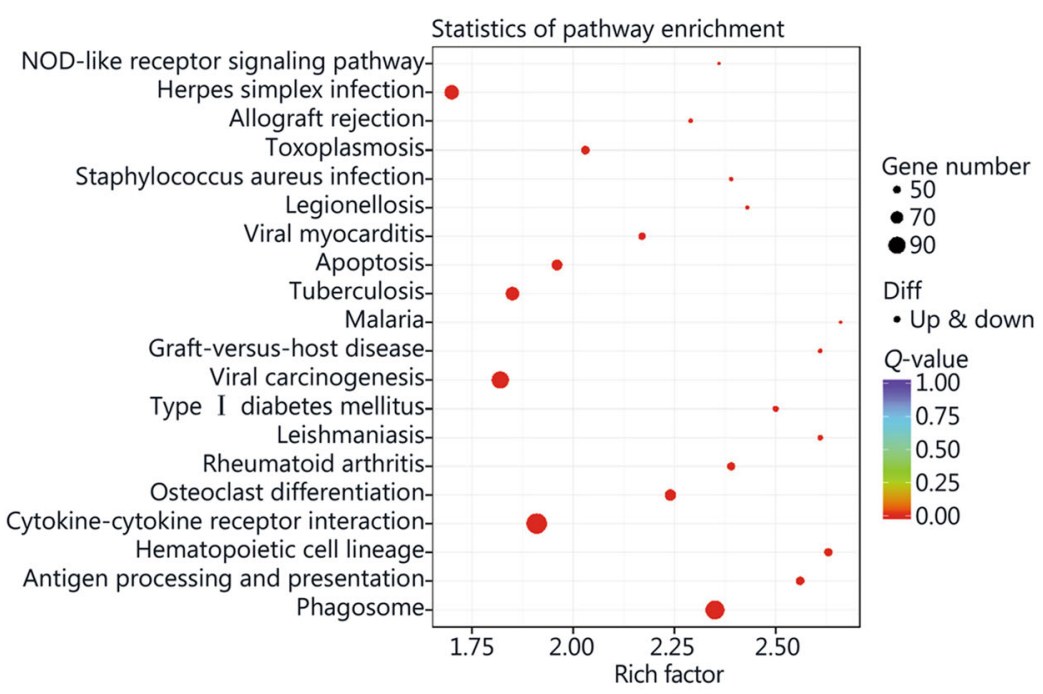

b

Fc epsilon RI signaling pathway-

Statistics of pathway enrichment

Protein processing in endoplasmic reticulum-

Beta-Alanine metabolism-

Oocyte meiosis-

Legionellosis

Homologous recombination-

Dorso-ventral axis formation

Circadian rhythm

Huntington's disease-

Ribosome-

Regulation of actin cytoskeleton

Cardiac muscle contraction-

TNF signaling pathway-

Basal transcription factors-

Arrhythmogenic right ventricular cardiomyopathy (ARVC)

Progesterone-mediated oocyte maturation-

Notch signaling pathway-

Alzheimer's disease-

Oxidative phosphorylation-

Parkinson's disease

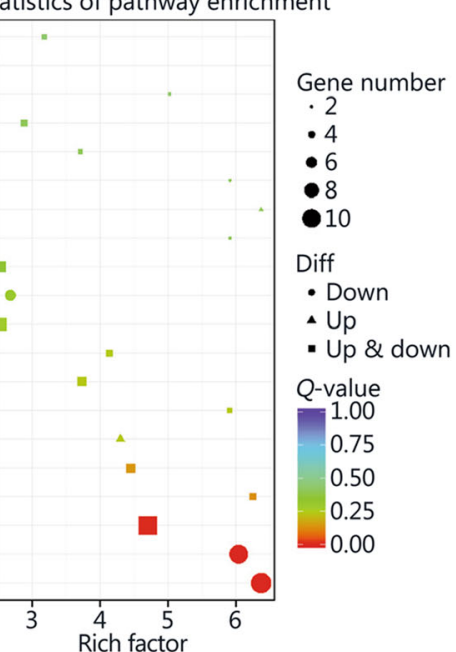

C

Statistics of pathway enrichment

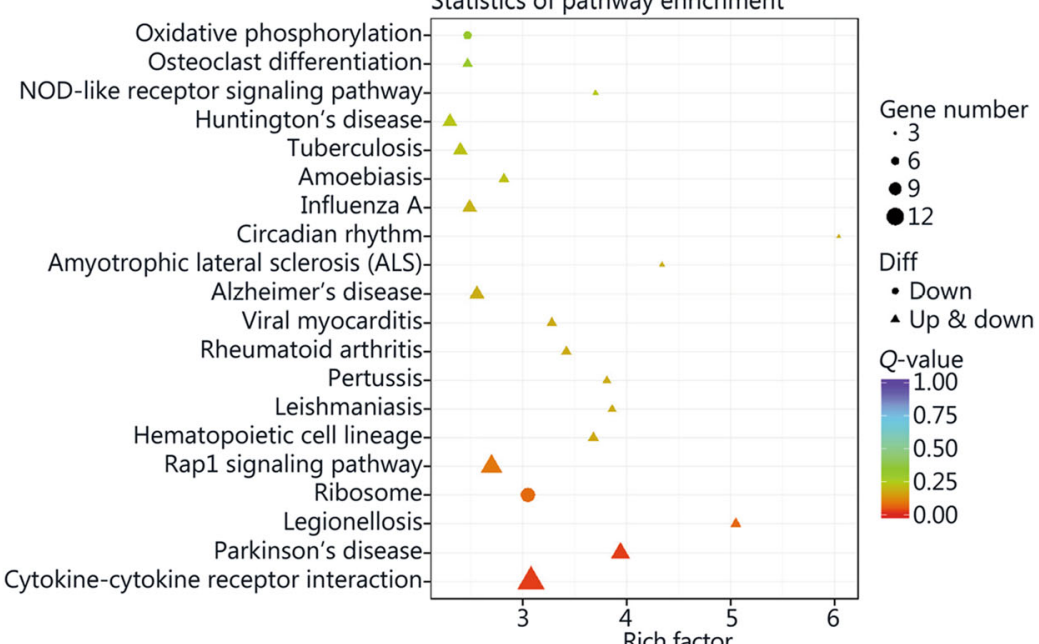

Fig. 9 (See legend on next page.) 
(See figure on previous page.)

Fig. 9 Kyoto Encyclopedia of Genes and Genomes (KEGG) analyses of significant differentially expressed (DE) genes identified by comparing before and after M. tuberculosis infection or NiuBeiXiaoHe (NBXH) treatment. The abscissa is the enrichment factor, indicating the ratio of the gene proportion annotated to the pathway in the DE genes to that annotated to the pathway in all genes. The larger the enrichment factor, the more significant the enrichment level of DE genes in this pathway. a Tuberculosis (TB) model group vs. normal control group. b JieHeWan (JHW) vs. TB model group. c NBXH vs. TB model group

results indicate that $\mathrm{NBXH}$ treatment mainly plays an anti-TB role by regulating the host immune response and inflammatory response, and its mechanism of action is different from that of JHW, NBXH is more targeted for $\mathrm{TB}$ and may be more effective in restoring immune damage caused by TB. The results in Table 1 and Table 2 also support this view. Most of the top 30 significantly up- or down-regulated $\mathrm{DE}$ genes were recovered by NBXH treatment after $M$. tuberculosis infection, which is consistent with only approximately one-third of the significantly up- or down-regulated DE genes being restored after JHW treatment, indicating that NBXH treatment has a more significant reparative effect on cell damage caused by $M$. tuberculosis infection, which may be due to the different components, targets and mechanisms of action of these two kinds of traditional Chinese medicine prescriptions. In addition, at present, it is difficult to evaluate the effectiveness of traditional Chinese medicine in animal experiments. The theoretical systems of traditional Chinese medicine and Western medicine are totally different. It is usually difficult to obtain significant differences in the evaluation of traditional Chinese medicine by the efficacy evaluation indexes used in Western medicine. The cell damage repair function of NBXH identified by the gene expression profile may also be used as one of the indicators for efficacy evaluation of traditional Chinese medicine. This is because the recovery of significant changes in gene expression caused by pathogenic bacteria will inevitably result in a good change in the phenotype of host cells, such as the pathological damage to lung tissue in the NBXH group being relatively light. In the future, we will further study the efficacy evaluation and modernization of traditional Chinese medicine to provide ideas and experience.

The mechanism of action of NBXH is relatively complicated, involving changes in the expression of many different functional genes. The genes listed in Tables 1 and 2 may be the targets of NBXH and may also be important links in its signal transduction pathway. We analyzed the changes and functions of several representative DE genes to partially clarify the mechanism of action of NBXH. (1) The genes acting on signaling pathways and regulation include the Rho gene, which is a member of the group of guanosine triphosphate (GTP)-binding proteins called Rho GTPases because of their GTPase activity. Rho is a subfamily member of a small $G$ protein superfamily and a member of the Ras superfamily, functions as a key molecule in multiple signal transduction pathways in cells and plays a key regulatory role in a series of cell processes. NBXH-mediated upregulation may play an important role in anti-TB activity in the immune response by affecting the biological processes of activation, proliferation, adhesion, and migration of immune cells such as $\mathrm{T}$ lymphocytes, B lymphocytes, monocytes, and macrophages [45, 46]. Rap guanine nucleotide exchange factor 5 (Rapgef5) plays an important role in the process of multicellular differentiation by regulating the Wnt signaling pathway [47]. Gm38394 has transcription factor activity and can control the transcription and expression of DNA by binding with specific DNA sequences, and NBXH can recover and increase its expression to improve the activity of cells. (2) The genes regulating the immune response and inflammatory response include the Mapk14 gene, which is a member of the mitogen-activated protein kinase (MAPK) family. Its functions include extracellular domain activity and protein binding, regulating cell growth, differentiation, oxidative stress, the inflammatory response, and other important cellular physiological/ pathological processes [48, 49]. NBXH upregulates Mapk14 expression, regulates the immune response during $M$. tuberculosis infection, induces BAX translocation and apoptosis, enhances TNF expression, limits $M$. tuberculosis, and exerts an anti-TB effect [50]. The protein Crispld2 can bind to LPS, and LPS binds to TLR4 receptors, thereby exerting a feedback regulation mechanism, and its up-regulated expression can reduce the release of inflammatory mediators (such as TNF- $\alpha$ and IL-6), systemic inflammation, and the degree of lung injury [51]. ZC3H11A is a stress-induced protein. Currently, some viruses (such as adenovirus, influenza virus, HIV, and herpes simplex virus) are known to rely on the expression of this gene. NBXH treatment can significantly reduce the expression of this gene [52]. On the one hand, ZC3H11A plays an anti-inflammatory role and may protect cells from damage. On the other hand, it may also contribute to antiviral infection in the body. C5aR1 is the receptor of the complement factor C5a. C5a regulates various biological processes, such as immune inflammation, opsonization, and cell lysis, through the C5aR1 pathway [53]. NBXH significantly downregulates the expression of this gene and may also protect cells from inflammatory damage. (3) The genes regulating cell differentiation and proliferation include the Srp54a gene, 
which is a 54-kDa signal recognition particle [54] with GTP binding and catalytic activity that mediates protein recognition and transport and participates in the directional transfer of intracellular proteins. NBXH-mediated restoration of the genes down-regulated by $M$. tuberculosis infection plays an important role in cell differentiation and metabolism. (4) The genes regulating energy metabolism include the Slc5a1 gene, which is a glucose transporter [55]. NBXH increases Slc5a1 expression to promote maximal glucose influx into cells to generate energy and promote cell metabolism. Additionally, the anti-TB effect of many newly identified up- and downregulated genes, as well as the correlations between genes and between genes and proteins, have not been elucidated. Further research is needed to reveal more mechanisms of action and pathways.

\section{Conclusions}

In summary, NBXH had similar therapeutic effects as the patented Chinese drug preparation JHW, which is used in the clinic, in improving lung histopathology, reducing lung colony counts, and regulating the levels of cytokines. $\mathrm{NBXH}$ restored significant changes in gene expression caused by $M$. tuberculosis infection by regulating the expression of immune-related genes and activity of signaling pathways and repairing cell damage caused by $M$. tuberculosis infection. In this study, using a gene expression profile to observe the repair of injury caused by $M$. tuberculosis infection after drug treatment is proposed for the first time as an indicator for judging the efficacy of new drugs, which provides the experimental basis for the further development and clinical application of the compound NBXH. We will also study the combined effect of traditional Chinese medicine and chemical medicine to lay the experimental foundation for the establishment of a combined treatment regimen in the future.

\section{Abbreviations}

BP: biological process; CBA: cytometric bead array; CC: cellular component; CFUs: colony-forming units; CSF: colony-stimulating factor; DE: differentially expressed; GM-CSF: granulocyte-macrophage colony-stimulating factor;

GO: gene ontology; IFN-ץ: interferon gamma; JHW: JieHeWan; IL: interleukin; KEGG: Kyoto Encyclopedia of Genes and Genomes; $M$.

tuberculosis: Mycobacterium tuberculosis; MDR-TB: multidrug-resistant tuberculosis; MF: molecular function; NBXH: NiuBeiXiaoHe; NK: natural killer; TB: tuberculosis; Th-1: helper T lymphocytes-1; Th-2: helper T lymphocytes-2; TNF: tumor necrosis factor

\section{Supplementary Information}

The online version contains supplementary material available at https://doi. org/10.1186/s40779-021-00324-5.

Additional file 1: Fig. S1 Heatmap of the differential expression profile of genes compared between the Tuberculosis (TB) model group and normal control group, JieHeWan (JHW) group or NiuBeiXiaoHe (NBXH) group. The abscissa represents the sample name and sample clustering result, and the ordinate represents the differential gene and gene clustering result. Different columns represent different samples, and different rows represent different genes. The color represents the gene expression level $\left(\log _{10}\right)$ in the sample. The green dots represent downregulated DE genes, the red dots represent up-regulated DE genes, and the black dots represent nondifferentially expressed genes. a TB model group (T01,T02,T03) vs. normal control group (T13,T14,T15). b JHW (T04,T05,T06) vs. TB model group. c NBXH $(T 07, T 08, T 09)$ vs. TB model group.

Additional file 2: Fig. S2 Volcano plot of the differential expression profile of genes compared between the Tuberculosis (TB) model group and normal control group, JieHeWan (JHW) group or NiuBeiXiaoHe (NBXH) group. Each point in the differential expression volcano plot represents a gene, the abscissa represents the logarithm of the difference in the expression level of a gene between two samples, and the ordinate represents the negative logarithm of the $P$-value. The green dots represent down-regulated DE genes, the red dots represent up-regulated $D E$ genes, and the black dots represent nondifferentially expressed genes. a TB model group vs. normal control group. b JHW vs. TB model group. c NBXH vs. TB model group.

\section{Acknowledgments}

We thank all funders for supporting this study.

\section{Authors' contributions}

LYD participated in animal experiments, collected and analyzed the data and was a major contributor in the writing of the manuscript. YL was in charge of animal experiments and analyzed the data. WPG, JM, JW, LW, and ZXJ participated in animal anatomy work. YX and HL participated in the immunoassay. YML performed the pathology experiments. JL provided NBXH. XQW and YZ contributed to the study design and data analyses and revised the manuscript. All authors read and approved the final manuscript.

\section{Funding}

This study was supported by a grant from the Beijing G20 engineering innovation research project (NO. Z171100001717010).

\section{Availability of data and materials}

The datasets used during the current study are available from the corresponding author upon reasonable request.

\section{Declarations}

\section{Ethics approval and consent to participate}

The experiments involving animals were approved and conducted by the Animal Ethical Committee of the 8th Medical Center of the Chinese PLA General Hospital, and mouse care met the standards of the Experimental Animal Regulation Ordinances defined by the China National Science and Technology Commission.

\section{Consent for publication}

Not applicable.

\section{Competing interests}

The authors declare that they have no competing interests.

\section{Author details}

${ }^{1}$ Tuberculosis Prevention and Control Key Laboratory, Beijing Key Laboratory of New Techniques of Tuberculosis Diagnosis and Treatment, Tuberculosis Research Institute, the 8th Medical Center, Chinese PLA General Hospital, Beijing 100091, China. ${ }^{2}$ HeBei North University, Zhangjiakou 075000, China. ${ }^{3}$ Clinical Laboratory, the 8th Medical Center, Chinese PLA General Hospital, Beijing 100091, China. ${ }^{4}$ Pathology Department, the 8th Medical Center, Chinese PLA General Hospital, Beijing 100091, China. ${ }^{5}$ Guangdong Qifang Pharmaceutical Co., Ltd, Guangzhou 510075, China.

Received: 28 May 2020 Accepted: 26 April 2021

Published online: 02 June 2021

\section{References}

1. World Health Organization. Global tuberculosis report. 2020. https://www. who.int/teams/global-tuberculosis-programme/data. Accessed 22 Jan 2021. 
2. Zhao GQ, Ren B, Dong XP, Tong TT, Zhang LL, Xiang H. The research progress of chuanbeimu. Pharma Clin Chin Med. 2012;3(6):59-64 doi: cnki: sun:Iczy.0.2012-06-023.

3. He XR, Wang XX, Fang JC, Zhao ZF, Huang $L H$, Guo H, et al. Bletilla striata: medicinal uses, phytochemistry and pharmacological activities. J Ethnopharmacol. 2017;195(2017):20-38. https://doi.org/10.1016/.j.jp.2016.11.026.

4. National Pharmacopoeia Commission. Pharmacopoeia of the people's republic of China (I). Beijing Chin Med Sci Tech. 2015;36,72,103,224,277.

5. Wang XM. The study on the antibacterial effect of Niubeixiaohe of traditional Chinese medicine in vitro and in vivo experiments. Nanjing Univ Chin Med. 2014

6. Li GM. Study on the mechanism of NiuBeiXiaoHe extract against Mycobacterium tuberculosis. Nanjing Univ TCM. 2017;22.

7. Ling $Y B$, Liang $Y$, Wang $X M$, Wang L, Zhang JX, Yang YR, et al. The study on the targets of the antituberculosis effect of traditional Chinese medicine Niubeixiaohe extracts. Chin J Antituberc. 2016;38(1):17-22. https://doi.org/1 0.3969/.issn.1000-6621.2016.01.006.

8. Chen D. The research on the immunology and general pharmacology of the anti-tuberculosis Chinese medicine preparations Niubeixiaohe. Nanjing Univ Chin Med. 2014;38.

9. Zhang JG, Wang $L P$, Sun $Y$. Jiehewan adjuvant treatment of tuberculosis. Jilin J TCM. 2016;36(8):790-2. https://doi.org/10.13463/j.cnki.jzyy.2016.08.011.

10. Liu X, Wu XQ. Research progress of traditional Chinese medicine in treatment of multi-drug resistant pulmonary tuberculosis. Chin J Antituberc. 2016;38(1):53-6. https://doi.org/10.3969/j.issn.1000-6621.2016.01.012.

11. Yang QS, Yang $Y$, Wang XP, Chen $X$, et al. Research progress of Jiehewan in treating tuberculosis. Eval Drug Use Chin. 2017;17(4):571-6. https://doi.org/1 0.14009/j.issn.1672-2124.2017.04.051

12. Liang $Y$, Wang $X M$, Song JY, Wang $L$, Chen $D$, Yang $Y R$, et al. Therapeutic effects of traditional Chinese medicine Niubeixiaohe in mouse tuberculosis models. J Ethnopharmacol. 2017;195(2017):318-23. https://doi.org/10.1016/j. jep.2016.11.037.

13. Li RX, Liu XZ, Tang ZJ, Xian QY, Wang Y, Zhang J. Establishment of mouse model of acute infection with mycobacterium tuberculosis. J Pub Health and Prev Med. 2007;18(5):12-4. https://doi.org/10.3969/j.issn.1006-2483.2007. 05.004 .

14. Wang YP, Ye PL, Zhang CT, Lu RS, Lu ZF, Liu ZG. Effect of FeiLaoKang on the expression of caspase- 3 and IL-2 in mouse model of rifampin-resistant Mycobacterium tuberculosis. Mod TCM. 2015;35(5):146-50. https://doi.org/1 0.13424/j.cnki.mtcm.2015.05.057.

15. Xiang ZG, Lin SZ, Dong N, Yuan W, Xu YF, Qin C. Bacterial load and pathological changes in the spleen and lung of $\mathrm{M}$. tuberculosis hematogenous infected mice. Chin J Comp Med. 2011;21(8):66-8. https:// doi.org/10.3969/j.issn.1671-7856.2011.08.017.

16. Wu SR, Cai R, Li S, Shen K, Jia BZ, Li FR. Effects of Huangkuisu on killing and suppressing mycobacterium tuberculosis. J TCM Univ Hunan. 2011;31(11): 19-23. https://doi.org/10.3969/j.issn.1674-070X.2011.11.006.019.05.

17. Lu ZF, Ye PL, Chen XP, Lu RS, Zhang CT, Zhang FH. The effect of Fei-laokang on organ coefficient of rat with isoniazid resistant tuberculosis. Lishizhen Med Res. 2015;26(11):2564-5 doi: cnki:sun:szgy.0.2015-11-002.

18. Kang J, Wang LM, Wang P, Zhao Y, Zhang W, Han WD, et al. Establishment of a mouse model of infection with rifampicin-resistant mycobacterium tuberculosis. Acta Lab Anim Sci Sin. 2011;19(4):320-3. https://doi.org/10.3 969/j.issn.1005-4847.2011.04.011.

19. Jiang $\mathrm{S}$. The study on the therapeutic effects of DNA vaccines in a murine model of mycobacterium tuberculosis infection. Chongqing Med Univ. 2004;93.

20. Jiang JQ, Huang WP, Yan J, Li LW. Observation of anti-tuberculosis effect in Guinea pigs with Jiehewan. Chin J Zoonos. 2009;25(1):89-91. https://doi. org/10.3969/j.issn.1002-2694.2009.01.025

21. Thada S, Ponnana M, Sivangala R, Joshi L, Alasandagutti M, Ansari MS, et al. Polymorphisms of IFN- $-\mathrm{(}(+874 \mathrm{~A} / \mathrm{T})$ and IL-12 (+1188A/C) in tuberculosis patients and their household contacts in Hyderabad, India. Hum Immunol. 2016;77(7):559-65. https://doi.org/10.1016/j.humimm.2016.04.016.

22. Yang CY, Hu JC, Liu YW, Ma Y, Yang LY. Effect of vitamin D combined with anti- tuberculosis drugs on the serum levels of IL-1 and interferon gamma of elderly patients with spinal tuberculosis. Mod Biomed. 2017;17(9):1707-9 doi: cnki:sun:swcx.0.2017-09-029.

23. Xu ZC, Wang XY, Liu Z, Chen GZ, Chen G. The expression of IFN-Y, IL-12 and TNF-a in the serum and lesions of spinal tuberculosis patients and its effects on the disease. Mod Biomed. 2018;18(16):3057-61. https://doi.org/10.13241/ j.cnki.pmb.2018.16.012.
24. Song N, Li GH, Huang Q, Kong C, Wang HH, Xu Y. Construction and immunogenicity of A39 DNA vaccine against latent tuberculosis infection. $J$ Microbe Infect. 2015;10(1):19-27 doi: cnki:sun:wswg.0.2015-01-006.

25. Liu T, Xiang YG, Fan RH, Ma XH, Shi GM, Yu R, et al. Research progress on cytokines in pulmonary tuberculosis patients. Pract Prev Med. 2016;23(7): 894-6. https://doi.org/10.3969/j.issn.1006-3110.2016.07.040.

26. Su WL, Perng WC, Huang $\mathrm{CH}$, Yang $\mathrm{CY}, \mathrm{Wu} \mathrm{CP}$, Chen JH. Association of reduced tumor necrosis factor alpha, gamma interferon, and interleukin1 beta (IL-1beta) but increased IL-10 expression with improved chest radiography in patients with pulmonary tuberculosis. Clin Vaccine Immunol. 2010;17(2):223-31. https://doi.org/10.1128/CVI.00381-09.

27. Li ZH, Yang YH, Zhang HM, Tian YR, Liu X, Zhang GY, et al. Study on the relationship between the dynamic changes of cytokine levels and drug resistance in patients with pulmonary tuberculosis. Hebei Med. 2008;30(3): 332-3. https://doi.org/10.3969/j.issn.1002-7386.2008.03.039.

28. Zhang XX, Zhao CF. Determination and clinical significance of serum IL-4, IL-6 and TGF- $\beta$ in patients with pulmonary tuberculosis. J Pathog Biol. 2012; 7(3):195-6 doi: cnki:sun:zisc.0.2012-03-011.

29. Liu WS. The dynamic changes of IL-6 and IL-10 in serum of patients with tuberculosis and clinical significance. Nanchang Univ. 2010;16.

30. Beamer GL, Flaherty DK, Assogba BD, Stromberg P, Gonzalez-Juarrero M, de Waal MR, et al. Interleukin-10 promotes mycobacterium tuberculosis disease progression in CBA/J mice. J Immunol. 2008;181(8):5545-50. https://doi. org/10.4049/jimmunol.181.8.5545.

31. Hu YL. Research progress of tuberculosis cell immunity and cytokines. Qinghai Med. 2012;42(11):78-9 doi: cnki:sun:qhyz.0.2012-11-047.

32. LV Y, Liu WW, Wang C, Zhao H, Wang T, Bai L. The effect of infection with Mycobacterium tuberculosis H37Ra on T cells and the Th1/Th2 reaction in mice. J Pathog Biol. 2019;14(8):905-9 doi: cnki:sun:zisc.0.2019-08-008.

33. Van de Veerdonk FL, Teirlinck AC, Kleinnijenhuis J, Kullberg BJ, van Crevel R, van der Meer JW, et al. Mycobacterium tuberculosis induces IL-17A responses through TLR4 and dectin-1 and is critically dependent on endogenous IL-1. J Leukoc Biol. 2010;88(2):227-32. https://doi.org/10.1189/jb.0809550.

34. Lombard R, Doz E, Carreras F, Epardaud M, Le Vern Y, Buzoni-Gatel D, et al. IL-17RA in non-hematopoietic cells controls CXCL-1 and 5 critical to recruit neutrophils to the lung of mycobacteria-infected mice during the adaptive immune response. PLoS One. 2016;11(2):e0149455. https://doi.org/10.1371/ journal.pone.0149455.

35. Kozakiewicz L, Chen Y, Xu J, Wang Y, Dunussi-Joannopoulos K, Ou Q, et al. $B$ cells regulate neutrophilia during mycobacterium tuberculosis infection and BCG vaccination by modulating the interleukin-17 response. PLoS Pathog. 2013;9(7):e1003472. https://doi.org/10.1371/journal.ppat.1003472.

36. Okamoto YY, Umemura M, Yahagi A, O'Brien RL, Ikuta K, Kishihara K, et al. Essential role of IL-17A in the formation of a mycobacterial infectioninduced granuloma in the lung. J Immunol. 2010;184(8):4414-22. https://doi. org/10.4049/jimmunol.0903332.

37. Safar HA, Mustafa AS, Amoudy HA, El-Hashim A. The effect of adjuvants and delivery systems on Th1, Th2, Th17 and treg cytokine responses in mice immunized with mycobacterium tuberculosis-specific proteins. PLoS One. 2020;15(2):e0228381. https://doi.org/10.1371/journal.pone.0228381.

38. Torrado E, Cooper AM. IL-17 and Th17 cells in tuberculosis. Cytokine Growth Factor Rev. 2010;21(6):455-62. https://doi.org/10.1016/j.cytogfr.2010.10.004.

39. Sheng YC, Yuan L, Jiang WL, Zhao Q Xu B. Study on serum Th1 /Th2 cytokines levels and associated factors in pulmonary tuberculosis patients. Shanghai J Prev Med. 2016;28(3):157-60 doi: cnki:sun:shyi.0.2016-03-009.

40. Zhang TT, ChuanShang HX, Tang YC, Zhang KX, Rao X, Jiang TH. Therapeutic effect of interleukin-12 on murine infection with Mycobacterium tuberculosis through modulating Th1 and Th2 cytokine balance. Chin J Tuberc Respir Dis. 2000;23(6):358-60 doi: 0.3760/j:issn:1001-0939.2000.06.011.

41. Sun YH, Zhang R, Pei SL. Application of gene expression difference comparison to research of traditional Chinese medicine. Chin Prac Med. 2009:4(30):218-20. https://doi.org/10.3969/.jissn.1673-7555.2009.30.176.

42. Karim AF, Chandra P, Chopra A, Siddiqui Z, Bhaskar A, Singh A, et al. Express path analysis identifies a tyrosine kinase Src-centric network regulating divergent host responses to mycobacterium tuberculosis infection. J Biol Chem. 2011;286(46):40307-19. https://doi.org/10.1074/ jbc.M111.266239.

43. Mehta M, Rajmani RS, Singh A. Mycobacterium tuberculosis WhiB3 responds to vacuolar $\mathrm{pH}$-induced changes in mycothiol redox potential to modulate phagosomal maturation and virulence [J]. J Biol Chem. 2016;291(6):2888903. https://doi.org/10.1074/jbc.M115.684597. 
44. Liu Y, Zhang YL, Zhang XX, Li CY. Research progress of mycobacterium tuberculosis infection and immune escape mechanism. Chin J Microbiol Immunol. 2015;35(5):398-400. https://doi.org/10.3760/cmaj.jisn.0254-5101.2015.05.015.

45. Xu JD. Function of rho GTPases in innate immunity of kuruma shrimp and the induction and mechanisms of tyained innate immunity against virus in the shrimp. Shandong Univ. 2018;1.

46. Wang XJ, He YL. Research progress of rho kinases in immune cells and autoimmune diseases. Int J Immunol. 2020;43(1):83-7. https://doi.org/10.3 760/cma.jissn.1673-4394.2020.01.016.

47. Griffin JN, del Viso F, Duncan AR, Robson A, Hwang W, Kulkarni S, et al. RAPGEF5 regulates nuclear translocation of $\beta$-catenin. Dev Cell. 2018;44(2): 248-60. https://doi.org/10.1016/j.devcel.2017.12.001.

48. Zhou Y, Wang Q, Mark Evers B, Chung DH. Oxidative stress-induced intestinal epithelial cell apoptosis is mediated by p38 MAPK. Biochem Biophys Res Commun. 2006;350(4):860-5. https://doi.org/10.1016/j.bbrc.2006.09.103.

49. Duan YQ, Cheng WD, Du J, Zhu LM, Yang XY, Cheng YX, et al. Effect of Sijunzi decoction on SP/CCK and Mapk14 mRNA expression in rats with spleen Q deficiency. J Chin Med. 2014;37(4):656-60 doi: 1001-4454(2014)02-0656-05.

50. Yuan G, Bai Y, Zhang Y, Xu GY, Zhang JF. Data mining mycobacterium tuberculosis pathogenic gene transcription factors and their regulatory network nodes. Int J Genomics. 2018;2018(3):1-9. https:/doi.org/10.1155/2018/3079730.

51. Liu XF, Zhai JL, Li WF, Lin ZF. Protective effect of CRISPLD2 protein on acute respiratory disease syndrome induced by lipopolysaecharide in mice. J Navy Med. 2017;38(1):21-3. https://doi.org/10.3969/j.issn.1009-0754.2017.01.008.

52. Younis S, Kamel W, Falkeborn T, Wang H, Yu D, Daniels R, et al. Multiple nuclear-replicating viruses require the stress-induced protein $\mathrm{ZC} 3 \mathrm{H} 11 \mathrm{~A}$ for efficient growth. PNAS. 2018;115(16):3808-16.

53. Yan ZK, Qin ML, Liu YL, Li CR, Liu JJ, Xiao L. Developmental expression of C5aR1 in mouse brain and its effect on the differentiation of NSCs in vitro. Chin Soc Anatomy. 2012;111.

54. Zheng J, Dong HJ, Wang CX, Guan WJ, Li YQ. Research progress of targeting and translocation of proteins mediated by signal recognition particle in prokaryote. Acta Microbiol Sin. 2005;45(6):974-7. https://doi.org/1 0.3321/j.issn:0001-6209.2005.06.033.

55. Gao HF, Chen LY, Cheng CS, Chen H, Meng ZQ, Chen Z. SLC5A1 promotes growth and proliferation of pancreatic carcinoma via glucose-dependent AMPK/mTOR signaling. Cancer Manag Res. 2019;11(2019):3171-85. https:// doi.org/10.2147/CMAR.S195424

Ready to submit your research? Choose BMC and benefit from:

- fast, convenient online submission

- thorough peer review by experienced researchers in your field

- rapid publication on acceptance

- support for research data, including large and complex data types

- gold Open Access which fosters wider collaboration and increased citations

- maximum visibility for your research: over $100 \mathrm{M}$ website views per year

At $\mathrm{BMC}$, research is always in progress.

Learn more biomedcentral.com/submissions 